\title{
Dynamical stability of collisionless stellar systems and barotropic stars: the nonlinear Antonov first law
}

\author{
P. H. Chavanis
}

\author{
Laboratoire de Physique Théorique (UMR 5152 du CNRS), Université Paul Sabatier, 118 route de Narbonne, 31062 Toulouse, France \\ e-mail: chavanis@irsamc.ups-tlse.fr
}

Received 8 August 2005 / Accepted 22 December 2005

\section{ABSTRACT}

\begin{abstract}
We complete previous investigations on the dynamical stability of barotropic stars and collisionless stellar systems. A barotropic star that minimizes the energy functional at a fixed mass is a nonlinearly dynamically stable stationary solution of the Euler-Poisson system. Formally, this minimization problem is similar to a condition of "canonical stability" in thermodynamics. A stellar system that maximizes an $H$-function at fixed mass and energy is a nonlinearly dynamically stable stationary solution of the Vlasov-Poisson system. Formally, this maximization problem is similar to a condition of "microcanonical stability" in thermodynamics. Using a thermodynamical analogy, we provide a derivation and an interpretation of the nonlinear Antonov first law in terms of "ensembles inequivalence": a spherical stellar system with $f=f(\epsilon)$ and $f^{\prime}(\epsilon)<0$ is nonlinearly dynamically stable with respect to the Vlasov-Poisson system if the corresponding barotropic star with the same equilibrium density distribution is nonlinearly dynamically stable with respect to the Euler-Poisson system. This is similar to the fact that "canonical stability implies microcanonical stability" in thermodynamics. The converse is wrong in case of "ensembles inequivalence" which is generic for systems with long-range interactions like gravity. We show that criteria of nonlinear dynamical stability can be obtained very simply from purely graphical constructions by using the method of series of equilibria and the turning point argument of Poincaré, as in thermodynamics.
\end{abstract}

Key words. stellar dynamics - hydrodynamics - instabilities - gravitation - galaxies: structure - methods: analytical

\section{Introduction}

The dynamical stability of stars and galaxies is an important topic in astrophysics. A large number of results have been obtained in the past and are collected in Binney \& Tremaine (1987). Most of these results are based on a linearization of the Euler-Poisson and Vlasov-Poisson systems around a stationary solution and on the study of the evolution of the perturbation which is decomposed in normal modes $\mathrm{e}^{\sigma t} h_{\sigma}(\mathbf{x})$. In practice, one has to work out an eigenvalue equation determining the complex pulsation $\sigma$ and the corresponding eigenfunction $h_{\sigma}(\mathbf{x})$. This approach provides a condition of linear dynamical stability when $R_{\mathrm{e}}(\sigma) \leq 0$ for all $\sigma$ and a condition of instability otherwise. It has been found that a spherical stellar system with DF $f=f(\epsilon)$ and $f^{\prime}(\epsilon)<0$, where $\epsilon=v^{2} / 2+\Phi(\boldsymbol{r})$ is the individual energy, is linearly dynamically stable with respect to the Vlasov-Poisson system if the barotropic gas with the same equilibrium density distribution is linearly dynamically stable with respect to the Euler-Poisson system. This forms the Antonov first law (1960) and explains why the stability of stellar systems and gaseous systems are often treated in parallel although these systems are physically different (Binney \& Tremaine 1987).

In this paper, we shall tackle these dynamical stability problems by a different approach, using optimization principles.
These optimization principles provide conditions of nonlinear dynamical stability for barotropic stars and spherical galaxies. This is different from the thermodynamical stability of stellar systems (Padmanabhan 1990). In the thermodynamical context, we maximize the Boltzmann entropy $S_{\mathrm{B}}[f]=$ $-\int \frac{f}{m} \ln \frac{f}{m} \mathrm{~d} \boldsymbol{r} \mathrm{d} \boldsymbol{v}$ at fixed mass $M[f]$ and energy $E[f]$ in order to obtain the most probable equilibrium state $\left(f \sim \mathrm{e}^{-\beta m \epsilon}\right)$ of an $N$-body Hamiltonian system resulting from a "collisional" relaxation for $t \gg t_{\text {relax }}$, where $t_{\text {relax }} \sim(N / \ln N) t_{\mathrm{D}}$ is the Chandrasekhar relaxation time ( $t_{\mathrm{D}}$ is the dynamical time). In that case, the Boltzmann entropy is obtained from a combinatorial analysis (Ogorodnikov 1965). On the other hand, the maximization of any $H$-function $S[f]=-\int C(f) \mathrm{d} \boldsymbol{r} \mathrm{d} \boldsymbol{v}$, where $C$ is convex (i.e. $C^{\prime \prime}>0$ ), at fixed mass and energy determines a nonlinearly dynamically stable stationary solution $f=f(\epsilon)$ with $f^{\prime}(\epsilon)<0$ of the Vlasov-Poisson system which describes collisionless stellar systems for $t \ll t_{\text {relax }}$. Formally, these maximization problems are mathematically similar (the second one involving a larger class of functionals than the Boltzmann functional) but their physical content and interpretation are completely different: the first one refers to thermodynamics (collisional regime) while the second refers to dynamics (collisionless regime). However, due to their mathematical similarity, we can develop a thermodynamical analogy (Sect. 4.1) to analyze the nonlinear dynamical stability problem. In this 
analogy, the criterion of nonlinear dynamical stability for spherical stellar systems with $f=f(\epsilon)$ and $f^{\prime}(\epsilon)<0$ described by the Vlasov-Poisson system (Sect. 2.2) is similar to a criterion of thermodynamical stability in the microcanonical ensemble. We have also found that the criterion of nonlinear dynamical stability for barotropic stars described by the Euler-Poisson system (Sect. 2.1) is similar to a criterion of thermodynamical stability in the canonical ensemble. Using this thermodynamical analogy, we provide a derivation and an interpretation of the nonlinear Antonov first law (Sect. 4.2) in terms of "ensembles inequivalence": a spherical stellar system with $f=f(\epsilon)$ and $f^{\prime}(\epsilon)<0$ is nonlinearly dynamically stable with respect to the Vlasov-Poisson system if the corresponding barotropic star with the same equilibrium density distribution is nonlinearly dynamically stable with respect to the Euler-Poisson system. This is similar to the fact that "canonical stability implies microcanonical stability" in thermodynamics (see, e.g., Ellis et al. 2000; Bouchet \& Barré 2005). The converse is wrong in case of "ensembles inequivalence" which is generic for systems with long-range interactions like gravity. We show that criteria of nonlinear dynamical stability can be obtained very simply from purely graphical constructions by using the method of series of equilibria and the turning point argument of Poincaré (Sects. 4.3 and 4.5), like in thermodynamics (Sect. 3). This will open a connection between studies of dynamical and thermodynamical stability (Sect. 4.4). These results were sketched at the end of a preceding paper (Chavanis 2003a) but we think that it is important to re-discuss them here in a more logical way with amplification.

Optimization principles for self-gravitating systems have also been developed in connection with a notion of "generalized thermodynamics" (Tsallis 1988). It has been observed in many systems with long-range interactions (self-gravitating systems, two-dimensional turbulence, HMF model, ...) that coherent structures (galaxies, jets and vortices, clusters, ...) form spontaneously and persist for extremely long times (see Chavanis 2002c). Since these meta-equilibrium states, or quasistationary states (QSS), are not described by the Boltzmann distribution in general, some authors have suggested that they might be described by a generalized thermodynamics. In particular, it has been proposed to replace the Boltzmann entropy $S_{\mathrm{B}}[f]$ by the Tsallis entropy $S_{q}[f]=-\frac{1}{q-1} \int\left(f^{q}-\right.$ $f) \mathrm{d} \boldsymbol{r} \mathrm{d} \boldsymbol{v}$. In that respect, the coherent structures that quickly form in long-range systems (in the collisionless regime) are claimed to maximize the Tsallis entropy at fixed mass and energy (Boghosian 1996; Latora et al. 2002). In the case of self-gravitating systems, this generalized thermodynamical interpretation has been defended by Taruya \& Sakagami (2003). We think, however, that this approach stems from a mis-interpretation (Chavanis 2003a, 2005b). Metaequilibrium states such as galaxies result from an incomplete violent relaxation (Lynden-Bell 1967) and are stable stationary solutions of the Vlasov equation on the coarse-grained scale. The Tsallis functional $H_{q}[\bar{f}]=-\frac{1}{q-1} \int\left(\bar{f}^{q}-\bar{f}\right) \mathrm{d} \boldsymbol{r} \mathrm{d} \boldsymbol{v}$, expressed as a function of the coarse-grained DF $\bar{f}(\boldsymbol{r}, \boldsymbol{v})$, is a particular $H$-function in the sense of Tremaine et al. (1986), not a thermodynamical entropy. Its extremization at fixed mass and energy leads to stellar polytropes (Sect. 4.3) which are particular stationary solutions of the Vlasov equation that form simple mathematical models of spherical stellar systems. Furthermore, its maximization provides a condition of nonlinear dynamical stability for the Vlasov-Poisson system, not a condition of "generalized thermodynamical stability". The resemblance of the criterion of nonlinear dynamical stability with a criterion of "generalized thermodynamical stability" is essentially the mark of a thermodynamical analogy (Chavanis 2003a). In fact, the previous authors did not consider the Vlasov equation (which is capital in our interpretation) and tried to build a generalized thermodynamics in order to deal with observed non-Boltzmannian quasistationary states (QSS). Now, considering the Vlasov equation, there is no fundamental reason why the QSS resulting from incomplete violent relaxation should be described by stellar polytropes (Tsallis distribution). These form just a particular class of steady solutions of the Vlasov equation. In practice, it is extremely difficult to predict the metaequilibrium state reached by the system in case of incomplete relaxation and we are led to construct dynamically stable stationary solutions of the Vlasov equation so as to reproduce observations a posteriori (Binney \& Tremaine 1987). The Vlasov equation admits an infinite number of stationary solutions and only stable solutions must be considered. For spherical systems with $f=f(\epsilon)$ and $f^{\prime}(\epsilon)<0$, a powerful criterion of nonlinear dynamical stability is given by the above maximization principle. A connection with theories of incomplete violent relaxation that try to predict the DF reached by the system (Lynden-Bell 1967; Stiavelli \& Bertin 1987; Chavanis et al. 1996) is discussed in Sect. 5.

\section{Dynamical stability of stars and galaxies}

\subsection{Nonlinear dynamical stability of barotropic stars}

We consider a self-gravitating gaseous medium (star) described by the Euler-Poisson system

$$
\begin{aligned}
& \frac{\partial \rho}{\partial t}+\nabla \cdot(\rho \boldsymbol{u})=0 \\
& \frac{\partial \boldsymbol{u}}{\partial t}+(\boldsymbol{u} \cdot \nabla) \boldsymbol{u}=-\frac{1}{\rho} \nabla p-\nabla \Phi,
\end{aligned}
$$

$\Delta \Phi=4 \pi G \rho$.

We shall restrict ourselves to the case of a barotropic gas for which the equation of state $p=p(\rho)$ depends only on the density. With this assumption, the Euler-Poisson system is closed. The total energy of the fluid is

$\mathcal{W}[\rho, \boldsymbol{u}]=\int \rho \int^{\rho} \frac{p\left(\rho^{\prime}\right)}{\rho^{\prime 2}} \mathrm{~d} \rho^{\prime} \mathrm{d} \boldsymbol{r}+\frac{1}{2} \int \rho \Phi \mathrm{d} \boldsymbol{r}+\int \rho \frac{\boldsymbol{u}^{2}}{2} \mathrm{~d} \boldsymbol{r}$.

The first term is the work $-p(\rho) \mathrm{d}(1 / \rho)$ done in compressing it from infinite dilution, the second term is the gravitational energy and the third term is the kinetic energy associated with the mean motion.

It is straightforward to verify that the energy functional (4) is conserved by the Euler-Poisson system $(\dot{W}=0)$. Therefore, 
a minimum of $\mathcal{W}$ at fixed mass determines a stationary solution of the Euler-Poisson system which is (formally) nonlinearly dynamically stable in the sense of Holm et al. (1985). Physically, this means that a small perturbation from the minimum remains close (in some norm) to the minimum. We are led therefore to consider the minimization problem

$\operatorname{Min}\{\mathcal{W}[\rho, \boldsymbol{u}] \quad \mid M[\rho]=M\}$.

Cancelling the first order variations of Eq. (4) at fixed mass, we obtain $\boldsymbol{u}=\mathbf{0}$ and the condition of hydrostatic equilibrium

$\nabla p=-\rho \nabla \Phi$.

Therefore, extrema of $\mathcal{W}$ correspond to stationary solutions of the Euler-Poisson system (1)-(3). Combining the condition of hydrostatic equilibrium (6) and the equation of state $p=p(\rho)$, we get

$\int^{\rho} \frac{p^{\prime}\left(\rho^{\prime}\right)}{\rho^{\prime}} \mathrm{d} \rho^{\prime}=-\Phi$,

so that $\rho$ is a function of $\Phi$ that we note $\rho=\rho(\Phi)$. Considering now the second order variations, the condition of nonlinear $\mathrm{dy}$ namical stability is

$\delta^{2} \boldsymbol{W}=\frac{1}{2} \int \delta \rho \delta \Phi \mathrm{d} \boldsymbol{r}+\int \frac{p^{\prime}(\rho)}{2 \rho}(\delta \rho)^{2} \mathrm{~d} \boldsymbol{r} \geq 0$,

for all perturbations that conserve mass, i.e. $\int \delta \rho \mathrm{d} \boldsymbol{r}=0$. We note that the second integral in Eq. (8) can be written in a more conventional form by using $p^{\prime}(\rho) / \rho=-1 / \rho^{\prime}(\Phi)$ resulting from Eq. (7).

Let us consider explicitly some particular examples. For an isothermal gas $p=\rho \frac{k_{\mathrm{B}} T}{m}$, we find that

$\rho=A^{\prime} \mathrm{e}^{-\frac{m \Phi}{k_{\mathrm{B}} T}}$,

$\mathcal{W}=k_{\mathrm{B}} T \int \frac{\rho}{m} \ln \frac{\rho}{m} \mathrm{~d} \boldsymbol{r}+\frac{1}{2} \int \rho \Phi \mathrm{d} \boldsymbol{r}+\int \rho \frac{\boldsymbol{u}^{2}}{2} \mathrm{~d} \boldsymbol{r}$.

On the other hand, for a polytropic gas $p=K \rho^{\gamma}$, we have

$\rho=\left[\lambda-\frac{\gamma-1}{K \gamma} \Phi\right]^{\frac{1}{\gamma-1}}$,

$\mathcal{W}=\frac{K}{\gamma-1} \int\left(\rho^{\gamma}-\rho\right) \mathrm{d} \boldsymbol{r}+\frac{1}{2} \int \rho \Phi \mathrm{d} \boldsymbol{r}+\int \rho \frac{\boldsymbol{u}^{2}}{2} \mathrm{~d} \boldsymbol{r}$.

For $\gamma \rightarrow 1$, we recover the expressions (9) and (10) of the isothermal gas. The minimization problem (5) has been studied in Chavanis (2002a,b, 2003a) for an isothermal and a polytropic equation of state (the system can be self-confined or confined by a box or by an external pressure). It is shown that the condition of nonlinear dynamical stability coincides with the condition of linear dynamical stability (see also Appendix A). On the other hand, for isothermal systems, the energy functional $\mathcal{W}[\rho, \mathbf{0}]$ coincides with the Boltzmann free energy $F_{\mathrm{B}}[\rho]=E[\rho]-T S_{\mathrm{B}}[\rho]$ (see Chavanis 2002a, for details). Therefore, the condition of nonlinear dynamical stability with respect to the Euler-Poisson system is the same as the condition of thermodynamical stability in the canonical ensemble.

\subsection{Nonlinear dynamical stability of stellar systems}

We consider a stellar system (galaxy) described by the VlasovPoisson system

$\frac{\partial f}{\partial t}+\boldsymbol{v} \cdot \frac{\partial f}{\partial \boldsymbol{r}}+\boldsymbol{F} \cdot \frac{\partial f}{\partial \boldsymbol{v}}=0$

$\Delta \Phi=4 \pi G \int f \mathrm{~d} v$,

where $\boldsymbol{F}=-\nabla \Phi$ is the self-consistent gravitational field produced by the stars. The Vlasov description assumes that the evolution of the system is encounterless. This is a very good approximation for most stellar systems because the relaxation time is in general larger than the age of the universe by several orders of magnitude (Binney \& Tremaine 1987). The Vlasov equation conserves the total mass $M=\int f \mathrm{~d} \boldsymbol{r} \mathrm{d} \boldsymbol{v}$, the total energy $E=\frac{1}{2} \int f v^{2} \mathrm{~d} \boldsymbol{r} \mathrm{d} \boldsymbol{v}+\frac{1}{2} \int \rho \Phi \mathrm{d} \boldsymbol{r}$ and an infinite class of functionals called the Casimirs that are defined by $I_{h}=\int h(f) \mathrm{d} \boldsymbol{r} \mathrm{d} v$ for any function $h$. We shall be particularly concerned with a special class of Casimirs of the form

$S[f]=-\int C(f) \mathrm{d} \boldsymbol{r} \mathrm{d} \boldsymbol{v}$,

where $C$ is an arbitrary convex function, i.e. $C^{\prime \prime}>0$. Since $S$, $M$ and $E$ are conserved by the collisionless dynamics, the maximization problem

$\operatorname{Max}\{S[f] \quad \mid E[f]=E, M[f]=M\}$,

determines a nonlinearly dynamically stable stationary solution of the Vlasov-Poisson system. We note that $F[f]=E[f]-$ $T S[f]$, where $T$ is an arbitrary positive constant (we shall explain later why we restrict $T$ to positive values), is also conserved by the Vlasov equation. This is called a Casimir-Energy functional. Therefore, a minimum of $F$ at fixed mass $M$ also determines a nonlinearly dynamically stable stationary solution of the Vlasov-Poisson system. This criterion can be written

$\operatorname{Min}\{F[f] \quad \mid M[f]=M\}$.

Instead of minimizing $F$ at fixed mass, it proves more convenient to maximize the functional $J=S-\beta E$ at fixed mass, where $\beta=1 / T$. We shall often work with the functional $J$ in the following. Similar criteria of nonlinear dynamical stability have been introduced by Ellis et al. (2002) in the context of two-dimensional hydrodynamics described by the 2D Euler-Poisson system. The optimization problem (17) corresponds to the formal nonlinear dynamical stability criterion of Holm et al. (1985) and the optimization problem (16) corresponds to the refined dynamical stability criterion of Ellis et al. (2002). These criteria are in general not equivalent for systems with long-range interactions, and this is similar to a notion of "ensembles inequivalence" in thermodynamics (see Sect. 4.1). The criterion of Ellis et al. (2002) refines the nonlinear dynamical stability theorems of Arnold (1966) who first introduced the Casimir-Energy method in 2D hydrodynamics. Inspired by these studies, we have applied the same criteria (16) and (17) to study the nonlinear dynamical stability of collisionless stellar systems described by the Vlasov-Poisson system 
(Chavanis 2003a,b; Chavanis \& Sire 2004). Similar optimization principles have been introduced independently by Guo \& Rein $(1999,2001)$ in the mathematical literature. They first considered the minimization of a Casimir-Energy functional $E-S$ at fixed mass $M$ (Guo \& Rein 1999) and later obtained a refined criterion of nonlinear dynamical stability by minimizing the energy $E$ at fixed Casimir $S$ and mass $M$ (Guo \& Rein 2001). The first criterion is equivalent to minimizing $F$ at fixed mass (optimization problem (17)) and the second criterion is equivalent to maximizing $S$ at fixed energy $E$ and mass $M$ (optimization problem (16)).

Using Lagrange multipliers and writing respectively

$\delta S-\beta \delta E-\alpha \delta M=0$,

or

$\delta J-\alpha \delta M=0$,

the critical points of the variational problems (16) and (17) are both given by

$C^{\prime}(f)=-\beta \epsilon-\alpha$,

where $\epsilon=\frac{v^{2}}{2}+\Phi(\boldsymbol{r})$ is the energy of a particle by unit of mass. Since $C^{\prime}$ is a monotonically increasing function of $f$, we can inverse this relation to obtain

$f=F(\beta \epsilon+\alpha)$

where $F(x)=\left(C^{\prime}\right)^{-1}(-x)$. Therefore, the critical points of the optimization problems (16) and (17) are the same. They correspond to distribution functions of the form $f=f(\epsilon)$ which depend only on the energy. According to the Jeans theorem, these DF are particular stationary solutions of the Vlasov-Poisson system which form a sub-class of spherical galaxies (the general DF of spherical galaxies depends on energy $\epsilon$ and angular momentum $\boldsymbol{J}=\boldsymbol{r} \times \boldsymbol{v}$ or $\boldsymbol{J}=|\boldsymbol{r} \times \boldsymbol{v}|)$. From Eq. (20), we have the identity $f^{\prime}(\epsilon)=-\beta / C^{\prime \prime}(f)$ showing that $f(\epsilon)$ is a monotonic function. Since $f(\epsilon)$ should be decreasing at least for large energies, this imposes $\beta=1 / T>0$. Then, we conclude that $f^{\prime}(\epsilon)<0$ for all $\epsilon$. Inversely, any DF of the form $f=f(\epsilon)$ with $f^{\prime}(\epsilon)<0$ is a critical point of a certain Casimir $S$ of the form (15) at fixed mass and energy.

Furthermore, any DF of the form $f=f(\epsilon)$ with $f^{\prime}(\epsilon)<0$ which satisfies the optimization problems (16) or (17) is nonlinearly dynamically stable with respect to the Vlasov-Poisson system. The condition of nonlinear dynamical stability provided by the criterion (16) is

$$
\begin{array}{r}
\delta^{2} J=-\int C^{\prime \prime}(f) \frac{(\delta f)^{2}}{2} \mathrm{~d} \boldsymbol{r} \mathrm{d} \boldsymbol{v}-\frac{1}{2} \beta \int \delta \rho \delta \Phi \mathrm{d} \boldsymbol{r} \leq 0, \\
\forall \delta f \quad \mid \delta E=\delta M=0,
\end{array}
$$

and the condition of nonlinear dynamical stability provided by the criterion (17) is

$$
\begin{gathered}
\delta^{2} J=-\int C^{\prime \prime}(f) \frac{(\delta f)^{2}}{2} \mathrm{~d} \boldsymbol{r} \mathrm{d} \boldsymbol{v}-\frac{1}{2} \beta \int \delta \rho \delta \Phi \mathrm{d} \boldsymbol{r} \leq 0, \\
\forall \delta f \quad \mid \quad \delta M=0 .
\end{gathered}
$$

The first integral can be written in a more conventional form by using $f^{\prime}(\epsilon)=-\beta / C^{\prime \prime}(f)$. We note the importance of the first order constraints in the criteria (22) and (23). If condition (23) is satisfied for all perturbations that conserve mass, it is a fortiori satisfied for perturbations that conserve mass and energy. Therefore, condition (23) implies condition (22), but not the opposite. This is similar to the fact that canonical stability implies microcanonical stability in thermodynamics (but not the opposite). We conclude that the stability criterion (16) is more refined than the stability criterion (17): if a collisionless stellar system with $f=f(\epsilon)$ and $f^{\prime}(\epsilon)<0$ satisfies (16) or (17), it is nonlinearly dynamically stable; however, if it does not satisfy (17), it can be nonlinearly dynamically stable provided that it satisfies (16). This means that we can "miss" stable solutions if we use just the optimization problem (17) to construct stable stationary solutions of the Vlasov-Poisson system of the form $f=f(\epsilon)$ with $f^{\prime}(\epsilon)<0$. The problem (16) is richer and allows to construct a larger class of nonlinearly dynamically stable models of spherical galaxies. In particular, we shall see that stellar polytropes of index $3<n<5$ satisfy the criterion (16) but not the criterion (17).

\section{Thermodynamics of self-gravitating systems and series of equilibria}

\subsection{Classical particles}

Optimization problems of the form (16) and (17) were first studied in relation with the thermodynamics of stellar systems (Antonov 1962; Lynden-Bell \& Wood 1968) where $S$ is the Boltzmann entropy

$S_{\mathrm{B}}[f]=-\int \frac{f}{m} \ln \frac{f}{m} \mathrm{~d} \boldsymbol{r} \mathrm{d}$,

which can be obtained by a combinatorial analysis (Ogorodnikov 1965). In this thermodynamical context, the criterion (16) is a criterion of microcanonical stability and the criterion (17) is a criterion of canonical stability where $F_{\mathrm{B}}[f]=E[f]-T S_{\mathrm{B}}[f]$ is the Boltzmann free energy and $T$ the thermodynamical temperature. The critical points of $S_{\mathrm{B}}$ or $F_{\mathrm{B}}$ (with appropriate constraints) correspond to the mean-field Maxwell-Boltzmann distribution

$f=A \mathrm{e}^{-\beta m\left(\frac{v^{2}}{2}+\Phi\right)}$

where $\Phi(\boldsymbol{r})$ is related to $f$ via the Poisson Eq. (14). The optimization problems (16) or (17) determine the most probable state of the $N$-body system (statistical equilibrium state) resulting from a "collisional" relaxation. The microcanonical ensemble (fixed energy) is appropriate to isolated Hamiltonian systems like stellar systems. The relaxation towards statistical equilibrium is driven by two-body encounters (Binney \& Tremaine 1987) and the collisional evolution of stellar systems is described by the gravitational Landau equation which increases the Boltzmann entropy $S_{\mathrm{B}}$ (H-theorem) at fixed mass $M$ and energy $E$. Therefore, collisions (finite $N$ effects) eventually single out the Boltzmann distribution among all possible stationary solutions of the Vlasov equation. The canonical ensemble (fixed temperature) is appropriate to dissipative systems in contact with a heat bath (of non-gravitational 


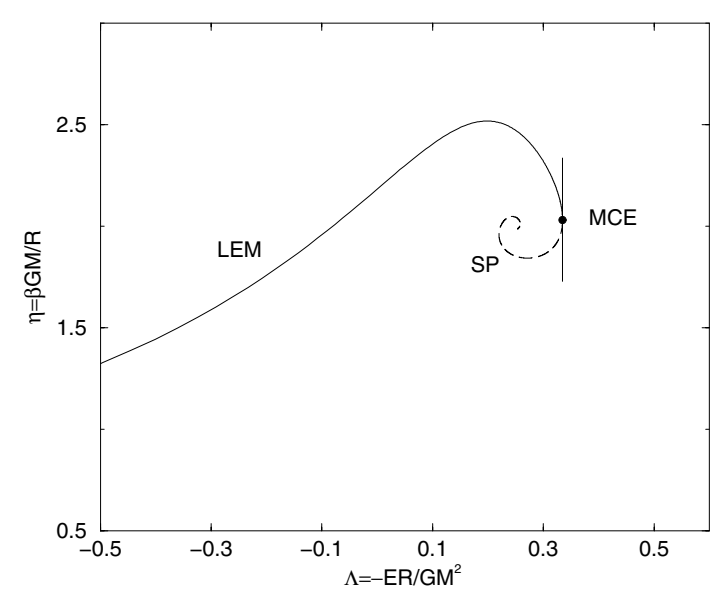

Fig. 1. Series of equilibria of isothermal stellar systems described by the Boltzmann entropy. The relevant dimensionless energy and dimensionless temperature are $\Lambda=-\frac{E R}{G M^{2}}$ and $\eta=\frac{\beta G M m}{R}$. The thermodynamic limit $N \rightarrow+\infty$ is such that these quantities remain fixed (Chavanis 2006b). In the microcanonical ensemble, the stable states (local entropy maxima LEM) are located on the full curve (microcanonical caloric curve). After the turning point of energy MCE, the states become unstable (saddle points SP of entropy).

origin) imposing its temperature, like in the model of selfgravitating Brownian particles introduced by Chavanis et al. (2002). The evolution of self-gravitating Brownian particles is described by mean-field Fokker-Planck equations (Kramers and Smoluchowski) which decrease the Boltzmann free energy $F_{\mathrm{B}}$ at fixed mass $M$ (Chavanis 2006b).

As is well-known, there is no global maximum of $S_{\mathrm{B}}$ (resp. global minimum of $F_{\mathrm{B}}$ ) but there can exist local maxima of $S_{\mathrm{B}}$ (resp. local minima of $F_{\mathrm{B}}$ ) if the system is confined within a box of radius $R$ and the energy (resp. temperature) is sufficiently high. These metastable states are long-lived because their lifetime scales as $\mathrm{e}^{N}$ so that they are of prime interest in astrophysics (Chavanis 2005a). The thermodynamical stability of the system can be settled by using the turning point method of Poincaré which was applied by Lynden-Bell \& Wood (1968) and Katz (1978) to classical self-gravitating systems. Since we shall apply this method in new situations (related to nonlinear dynamical stability problems), we first describe how this method works in thermodynamics referring to the previously quoted papers for technical details.

Suppose we want to maximize the Boltzmann entropy $S_{\text {B }}$ at fixed mass $M$ and energy $E$ (microcanonical stability). The parameter conjugate to the entropy $S_{\mathrm{B}}$ with respect to the energy $E$ is the inverse temperature $\beta=\left(\partial S_{\mathrm{B}} / \partial E\right)_{N, V}$. Therefore, if we plot the series of equilibria giving $\beta$ as a function of $-E$, we have the following results:

(i) a change of stability can occur only at a turning point where $E$ is an extremum $(\mathrm{d} \beta / \mathrm{d} E$ infinite);

(ii) a mode of stability is lost if the curve rotates clockwise and gained if the curve rotates anti-clockwise.

The series of equilibria of isothermal stellar systems described by the Boltzmann entropy is shown in Fig. 1 and forms a spiral. Now, we know that for $E$ sufficiently large, the solutions are stable (maxima of $S_{\mathrm{B}}$ at fixed $E, M$ ) because, in this limit,

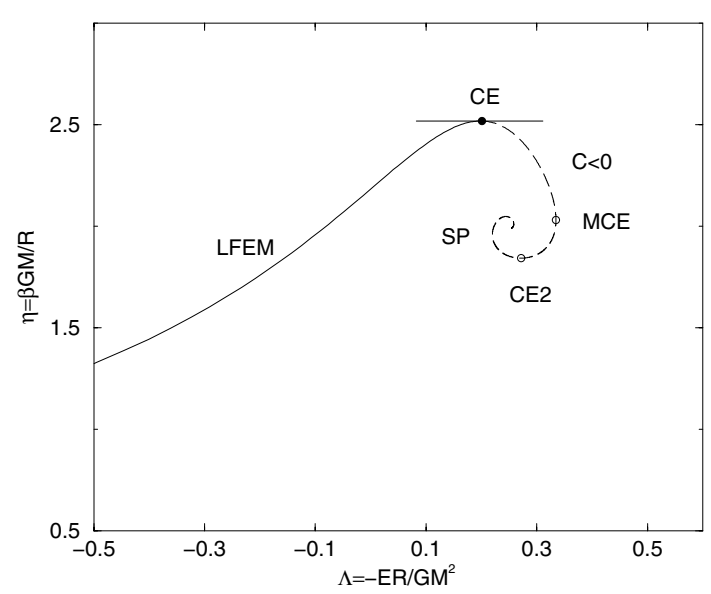

Fig. 2. Series of equilibria of isothermal stellar systems described by the Boltzmann free energy. In the canonical ensemble, the stable states (local free energy minima LFEM) are located on the full curve (canonical caloric curve). After the turning point of temperature $\mathrm{CE}$, the states become unstable (saddle points SP of free energy). The region of negative specific heats between CE and MCE is a domain of ensembles inequivalence: those states are stable in the microcanonical ensemble but unstable in the canonical ensemble.

self-gravity is negligible and the system behaves like an ordinary gas in a box. From point (i), we conclude that the whole upper branch until point MCE is stable and corresponds to local entropy maxima (LEM). This stable part of the curve defines the microcanonical caloric curve. At the first turning point of entropy MCE, the curve rotates clockwise so that a mode of stability is lost. As the curve spirals inward, more and more modes of stability are lost (at each vertical tangent) so that the states past MCE are unstable saddle points of entropy (SP).

Suppose now that we want to minimize the free energy $F$ at fixed mass (canonical stability). This is equivalent to maximizing the Massieu function $J=S-\beta E$ at fixed $M$. Now the parameter conjugate to $J$ with respect to $\beta$ is $-E=$ $(\partial J / \partial \beta)_{N, V}$. Therefore, by using the turning point criterion (properly adapted to the present case) ${ }^{1}$ we conclude that the upper branch of the spiral is stable in the canonical ensemble until the point $\mathrm{CE}$ and corresponds to local minima of free energy (FEM). This stable part of the curve defines the canonical caloric curve. At the first turning point of temperature $\mathrm{CE}$, the curve rotates clockwise so that a mode of stability is lost. As the curve spirals inward after CE, more and more modes of stability are lost (at each horizontal tangent) so that the states are saddle points of free energy (SP). We note that the states between $\mathrm{CE}$ and MCE are stable in the microcanonical ensemble (local maxima of entropy $S_{\mathrm{B}}$ at fixed $E$, $M$ ) but unstable in the canonical ensemble (saddle points of free energy $F_{\mathrm{B}}$ at fixed $M$ ). Such states have negative specific heats $C=(\mathrm{d} E / \mathrm{d} T)_{N, V}$. Negative specific heats are forbidden in the canonical ensemble but they are allowed in the microcanonical ensemble for systems with long-range interactions for which the energy is non-additive (Thirring 1970; Lynden-Bell \& Lynden-Bell 1977). Therefore, in this region,

1 We just need to rotate Fig. 2 by $90^{\circ}$ and plot $E$ as a function of $\beta$ to be in the conditions of application of (i) and (ii). 
the ensembles are inequivalent: the states with negative specific heats cannot be reached by a canonical description (they are unstable). Note that negative specific heats is a sufficient but not a necessary condition of canonical instability: for example, the states between MCE and CE2 have positive specific heats but they are canonically (and microcanonically) unstable.

\subsection{Self-gravitating fermions}

Another application of the turning point method where modes of stability can be lost and then re-gained has been studied by Chavanis (2002d) in the case of self-gravitating fermions. In that case, the thermodynamical potential is the Fermi-Dirac entropy

$S=-\frac{\eta_{0}}{m} \int\left\{\frac{f}{\eta_{0}} \ln \frac{f}{\eta_{0}}+\left(1-\frac{f}{\eta_{0}}\right) \ln \left(1-\frac{f}{\eta_{0}}\right)\right\} \mathrm{d} \boldsymbol{r} \mathrm{d} \boldsymbol{v}$

and its extremization at fixed mass and energy leads to the Fermi-Dirac distribution

$f=\frac{\eta_{0}}{1+\lambda \mathrm{e}^{\beta m\left(\frac{v^{2}}{2}+\Phi(\boldsymbol{r})\right)}}$,

where $\lambda>0$ and $\eta_{0}=(\mathrm{m} / \mathrm{h})^{3}$ is the maximum allowed value of the distribution function due to the Pauli exclusion principle. The form of the series of equilibria depends on the degeneracy parameter $\mu \equiv \eta_{0} \sqrt{512 \pi^{4} G^{3} M R^{3}}$. For a degeneracy parameter $\mu=10^{5}$, the series of equilibria is shown in Fig. 3. The stability of the configurations can be analyzed with the Poincare turning point argument. For large $E$, self-gravity is negligible with respect to thermal motion and the system coincides with a classical perfect gas in a box which is known to be thermodynamically stable. Thus, from (i) we conclude that the series of equilibria is microcanonically stable until MCE1. At that point, the tangent is vertical and the curve rotates clockwise so that a mode of stability is lost. However, a second turning point of energy appears at MCE2 and the curve turns anti-clockwise so that the mode of stability is re-gained. Therefore, the states between MCE1 and MCE2 are unstable saddle points of entropy at fixed mass and energy (SP) while the states before MCE1 and after MCE2 are maxima of entropy at fixed $E, M$. These stable states define the physical microcanonical caloric curve including the metastable states (local entropy maxima). The transition between local and global entropy maxima, the importance of metastable states and the notion of phase transitions (first order and zeroth order) between a gaseous phase and a condensed phase in the self-gravitating Fermi gas, and more generally in self-gravitating systems with a small-scale cut-off, are further discussed in Chavanis (2002d, 2005a).

2 The degeneracy parameter can also be written $\mu=$ $17.259 \ldots\left(R / R_{*}\right)^{3 / 2}$. It is proportional to the ratio, to the power $3 / 2$, between the system size $R$ and the size $R_{*}$ of a completely degenerate configuration (fermion ball at $T=0$ ) with mass $M$ (Chavanis \& Rieutord 2003). Roughly speaking, it is a measure of the importance of the small-scale regularization which is played here by the Pauli exclusion principle. Thus, $\epsilon=1 / \mu$ can be seen as an effective small-scale cut-off. In terms of the Planck constant, we have $1 / \mu \sim \hbar^{3}$ so that the classical limit $(\hbar \rightarrow 0)$ is recovered for $\mu \rightarrow+\infty$.

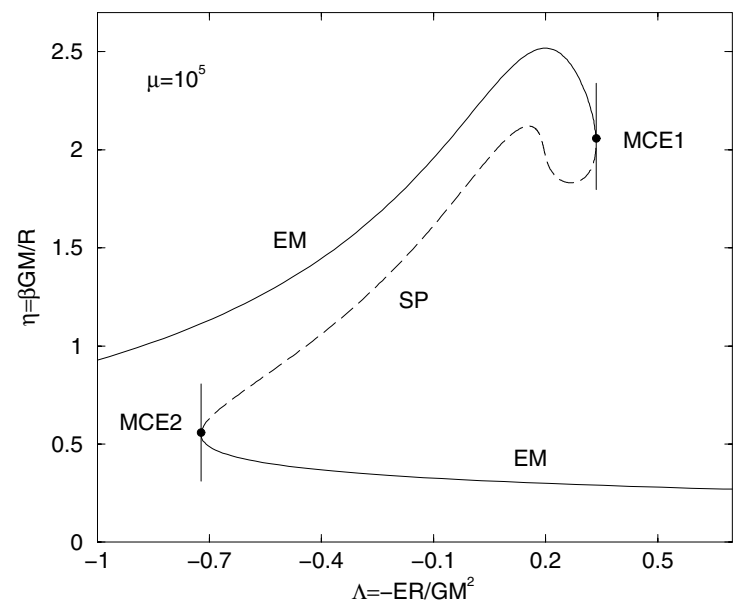

Fig. 3. Series of equilibria of self-gravitating fermions described by the Fermi-Dirac entropy for $\mu=10^{5}$. In the microcanonical ensemble, the stable states (entropy maxima EM) are located on the full curves. The upper branch corresponds to the "gaseous phase" and the lower branch to the "condensed phase". The mode of stability lost at MCE1 is re-gained at MCE2. The dashed curve corresponds to unstable saddle points of entropy.

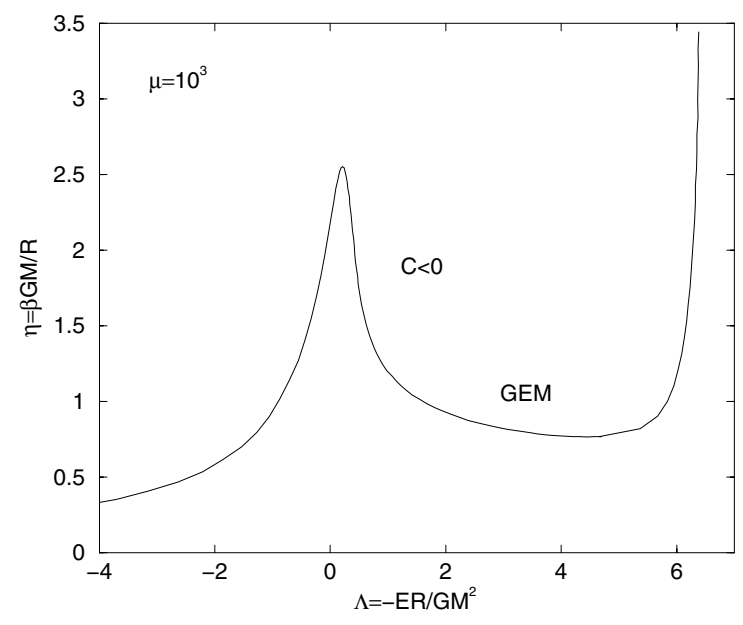

Fig. 4. Series of equilibria of self-gravitating fermions described by the Fermi-Dirac entropy for $\mu=10^{3}$. In the microcanonical ensemble, the full series of equilibria is stable and corresponds to global entropy maxima (GEM).

For a degeneracy parameter $\mu=10^{3}$, the series of equilibria is shown in Figs. 4 and 5. Since there is no turning point of energy, we conclude that the whole series of equilibria is stable in the microcanonical ensemble and corresponds to global entropy maxima (GEM) at fixed mass and energy. This forms the microcanonical caloric curve (see Fig. 4). Alternatively, in the canonical ensemble (see Fig. 5), the presence of turning points of temperature and the application of the Poincaré method (using the fact that for high temperatures the system is stable) implies that the series of equilibria is stable before CE1 and after CE2 (minima of free energy at fixed mass) and unstable in between (saddle points of free energy at fixed mass). The mode of stability lost at CE1 is re-gained at CE2. The stable states on the solid curves form the physical canonical caloric curve including the metastable states. By comparing Figs. 4 and 5 


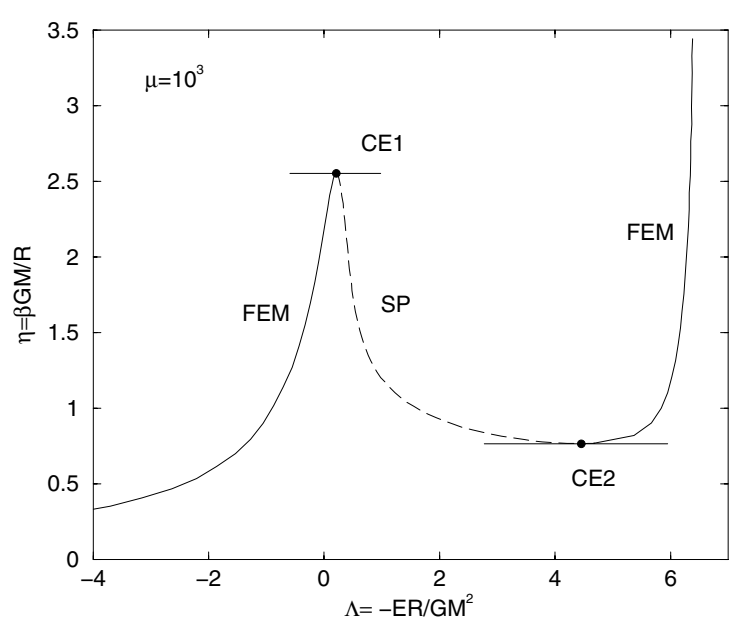

Fig. 5. Series of equilibria of self-gravitating fermions described by the Fermi-Dirac free energy for $\mu=10^{3}$. In the canonical ensemble, the stable states (minima of free energy FEM) are located on the full curves. The left branch corresponds to the "gaseous phase" and the right branch to the "condensed phase". The mode of stability lost at CE1 is re-gained at CE2. The dashed curve corresponds to unstable saddle points of free energy.

we note that the region of negative specific heats $C<0$ in the microcanonical ensemble is inaccessible (unstable) in the canonical ensemble where it is replaced by a phase transition.

Note, finally, that the Fermi-Dirac distribution (27) also arises in the theory of violent relaxation for collisionless stellar systems (Lynden-Bell 1967; Chavanis \& Sommeria 1998b). In that case, the exclusion principle is of dynamical origin since it is related to the conservation of the fine-grained DF by the Vlasov equation. Furthermore, the maximum phase-space density $\eta_{0}$ is related to the initial condition while $\eta_{0} \sim h^{-3}$ for fermions. Degeneracy effects (in the sense of Lynden-Bell) may be important in numerical simulations of violent relaxation (May \& van Albada 1984) and, possibly, in the core of elliptical galaxies (Stiavelli \& Bertin 1987; Chavanis \& Sommeria 1998b) and dark matter (Kull et al. 1996).

\section{Dynamical stability of stellar systems and barotropic stars}

\subsection{Thermodynamical analogy}

Formally, the criteria of nonlinear dynamical stability (16) and (17) for "collisionless" stellar systems are similar to criteria of thermodynamical stability for "collisional" selfgravitating systems (Sect. 3) but they involve a more general functional $S[f]=-\int C(f) \mathrm{d} \boldsymbol{r} \mathrm{d} \boldsymbol{v}$ than the Boltzmann entropy $S_{\mathrm{B}}[f]=-\int \frac{f}{m} \ln \frac{f}{m} \mathrm{~d} \boldsymbol{r} \mathrm{d} \boldsymbol{v}$. Furthermore, they have a completely different interpretation as they provide criteria of nonlinear dynamical stability for a stationary solution of the Vlasov equation while the maximization of the Boltzmann entropy at fixed mass and energy provides a condition of thermodynamical stability (most probable state) for the statistical equilibrium state of a $N$-body stellar system reached for $t \rightarrow+\infty$.

Due to the formal resemblance between nonlinear dynamical stability criteria and thermodynamical stability criteria, we can develop a thermodynamical analogy to investigate the nonlinear dynamical stability of collisionless stellar systems and use a vocabulary borrowed from thermodynamics. In this analogy, $S$ is similar to an entropy, $T=1 / \beta$ is similar to a temperature and $F$ is similar to a free energy (it is related to $S$ by a Legendre transform). The criterion (16) is similar to a condition of microcanonical stability and the criterion (17) is similar to a condition of canonical stability. Since canonical stability implies microcanonical stability (but not the converse), we recover the fact that condition (23) implies condition (22). The stability problems (16) and (17) can be studied by plotting the linear series of equilibria $\beta(E)$, which is similar to a caloric curve in thermodynamics. Due to the above thermodynamical analogy, the method of linear series of equilibria can be used to settle the nonlinear dynamical stability of a collisionless stellar system for a general functional $S$. According to the Poincaré theorem, a mode of stability is lost or gained at a turning point. Note that the slope $\mathrm{d} E / \mathrm{d} T$ (where $\beta=1 / T$ is the Lagrange multiplier associated with the energy) plays an important role because, according to the turning point argument, its change of sign may be the signal of an instability. The slope $C=\mathrm{d} E / \mathrm{d} T$ is similar to the specific heat in thermodynamics. We shall give some illustrations of these results in the following sections.

\subsection{The nonlinear Antonov first law}

For any stellar system with $f=f(\epsilon)$ and $f^{\prime}(\epsilon)<0$, there exists a corresponding barotropic star with the same equilibrium density distribution. Indeed, defining the density and the pressure by $\rho=\int f \mathrm{~d} v=\rho(\Phi), p=\frac{1}{3} \int f v^{2} \mathrm{~d} v=p(\Phi)$, and eliminating the potential $\Phi$ between these two expressions, we find that $p=p(\rho)$. Writing explicitly the density and the pressure in the form

$\rho=4 \pi \int_{\Phi}^{+\infty} f(\epsilon) \sqrt{2(\epsilon-\Phi)} \mathrm{d} \epsilon$

$p=\frac{4 \pi}{3} \int_{\Phi}^{+\infty} f(\epsilon)[2(\epsilon-\Phi)]^{3 / 2} \mathrm{~d} \epsilon$

and taking the gradient of Eq. (29), we obtain the condition of hydrostatic equilibrium (6).

As discussed in Sect. 2.2, the minimization problem (17) provides a sufficient (but not necessary) condition of nonlinear dynamical stability for a collisionless stellar system. To solve this minimization problem, we can first minimize $F[f]$ at fixed density $\rho(\boldsymbol{r})$ to obtain $f_{*}(\boldsymbol{r}, \boldsymbol{v})$. More precisely, $f_{*}$ is determined by $C^{\prime}\left(f_{*}\right)=-\beta\left(v^{2} / 2+\lambda(\boldsymbol{r})\right)$ where $\lambda(\boldsymbol{r})$ is the Lagrange multiplier associated with the specification of the density $\rho(\boldsymbol{r})$. Since $\delta^{2} F=(T / 2) \int C^{\prime \prime}\left(f_{*}\right)(\delta f)^{2} \mathrm{~d} \boldsymbol{r} \mathrm{d} v>0, f_{*}$ really is a minimum of $F$ at fixed $\rho(\boldsymbol{r})$ (note that fixing $\rho(\boldsymbol{r})$ automatically determines the potential energy $W=(1 / 2) \int \rho \Phi \mathrm{d} \boldsymbol{r}$ which can thus be treated as a constant). From the optimal distribution function $f_{*}$, we can compute the density $\rho=\int f_{*} \mathrm{~d} v=\rho(\lambda)$ and the pressure $p=\frac{1}{3} \int f_{*} v^{2} \mathrm{~d} \boldsymbol{v}=p(\lambda)$. Eliminating $\lambda(\boldsymbol{r})$ between these two expressions, we obtain the equation of state of a barotropic gas $p=p(\rho)$. This is the same equation of state as in equilibrium where $\lambda(\boldsymbol{r})=\Phi(\boldsymbol{r})+\alpha / \beta$ but the important point is 
that the relation $p=p(\rho)$ also holds for perturbations $\delta \rho$ around equilibrium. We can then express the functional $F[f]$ as a functional of the density $\rho(\boldsymbol{r})$ by setting $F[\rho]=F\left[f_{*}\right]$. The calculations are detailed in Appendix B (see also Sects. 7.7 and 7.8 of Chavanis 2003a) and the functional $F[\rho]$ can be finally written

$F[\rho]=\frac{1}{2} \int \rho \Phi \mathrm{d} \boldsymbol{r}+\int \rho \int^{\rho} \frac{p\left(\rho^{\prime}\right)}{\rho^{\prime 2}} \mathrm{~d} \rho^{\prime} \mathrm{d} \boldsymbol{r}$,

where $p(\rho)$ is the equation of state of the corresponding barotropic gas defined above. We are thus led to consider the minimization problem

$$
\operatorname{Min}\{F[\rho] \quad \mid M[\rho]=M\}
$$

Now, we observe that the functional (30) coincides with the energy functional (4) of a barotropic gas with $\boldsymbol{u}=\mathbf{0}$. We come therefore to the following conclusion: for a given stellar system with $f=f(\epsilon)$ and $f^{\prime}(\epsilon)<0$, if we know that the corresponding barotropic star is nonlinearly dynamically stable with respect to the Euler-Poisson system, then it is a minimum of $\mathcal{W}[\rho, \boldsymbol{u}]$, hence of $F[\rho]$ (at fixed mass). Therefore, the stellar system satisfies the criterion (17) so it is nonlinearly dynamically stable with respect to the Vlasov-Poisson system. This leads to a nonlinear generalization of the Antonov first law ${ }^{3}$ : "a spherical stellar system with $f=f(\epsilon)$ and $f^{\prime}(\epsilon)<0$ is nonlinearly dynamically stable with respect to the Vlasov-Poisson system if the corresponding barotropic gas with the same equilibrium density distribution is nonlinearly dynamically stable with respect to the Euler-Poisson system". However, the reciprocal is wrong in general. A stellar system can be nonlinearly dynamically stable according to the "microcanonical" criterion (16) while it does not satisfy the "canonical" criterion (17) so that the corresponding barotropic star is dynamically unstable. Due to the thermodynamical analogy, the nonlinear Antonov first law for collisionless stellar systems has the same status as the fact that: "canonical stability implies microcanonical stability in thermodynamics". The converse is wrong in case of "ensembles inequivalence" which is generic for systems with longrange interaction such as gravity. To the point of view of their nonlinear dynamical stability, the crucial difference between stars and galaxies is that, for galaxies, both $S$ and $E$ are individually conserved by the Vlasov equation while in the case of stars only the total energy $\mathcal{W}$ is conserved by the Euler equations. Therefore, the most refined stability criterion for spherical galaxies is (16) while the most refined stability criterion for stars is (5), which is included in (16). This is the intrinsic reason why a spherical galaxy can be stable even if the corresponding barotropic star is unstable. Therefore, the set of stable spherical galaxies is larger than the set of stable barotropic stars. Similarly, in thermodynamics, the set of stable microcanonical equilibria is wider than the set of stable canonical equilibria (it includes in particular states with negative specific heats) because there is an additional constraint in the problem: the energy.

\footnotetext{
3 This result has been obtained independently in Chavanis (2003a) and Rein $(2003,2005)$.
}

\subsection{Application to polytropes}

It is interesting to consider the application of these results to the case of polytropes. Stellar polytropes extremize the Casimir functional

$S_{q}=-\frac{1}{q-1} \int\left(f^{q}-f\right) \mathrm{d} \boldsymbol{r} \mathrm{d} \boldsymbol{v}$,

at fixed mass and energy. This leads to the class of distribution functions

$f=\left[\mu-\frac{\beta(q-1)}{q} \epsilon\right]_{+}^{\frac{1}{q-1}}$,

with the convention that $f=0$ when the term in brackets becomes negative. The usual polytropic index $n$ is related to the parameter $q$ by $n=\frac{3}{2}+\frac{1}{q-1}$. We can now plot the series of equilibria $\beta(E)$ issued from the optimization problems (16) and (17) with the polytropic functional (32). The equations determining these curves (for various index $n$ ) have been given in previous papers (Chavanis 2003a; Chavanis \& Sire 2004) so we shall only describe the results and present a "gallery" of caloric curves. We recall that the system is confined within a box of radius $R$ so that the series of equilibria is essentially formed by incomplete polytropes (represented in solid line) which are held by the box so that $\rho(R)>0$. The series of equilibria can be parameterized by the density contrast $\mathcal{R}=\rho(0) / \rho(R)$ between center and edge. The terminal point of the series of equilibria corresponds to the first complete polytrope (represented by a bullet) for which the density drops to zero precisely at the box radius $\rho(R)=0$ (if $n<5$ ). In the present context, this is the most important point in the series of equilibria because it connects the branch of incomplete polytropes to the branch of complete polytropes (represented by a dashed line) for which the density drops to zero at $R_{*}<R$ (if $n<5$ ). These are the physical structures in astrophysics since they are not affected by an artificial box. We thus have to investigate the stability of the first complete polytrope (bullet) to determine the stability of all the complete polytropes ${ }^{4}$ which are of physical relevance. To that purpose, we just need to investigate the presence of turning points in the series of equilibria. Therefore, our method is very simple and very general. It just requires to solve the first order variations of the optimization problems (16) and (17) and use the turning point argument to obtain powerful results of nonlinear dynamical stability for barotropic stars and spherical galaxies.

For $n<3$ the series of equilibria $\beta(E)$ for polytropes is monotonic (see Fig. 6) so that, according to the Poincaré criterion, all the polytropes of the series are maxima of $S$ (at fixed $E$, $M$ ) and minima of $F$ (at fixed $M$ ). In our thermodynamical analogy, this corresponds to a situation of ensembles equivalence. These results imply that, for $n<3$, both gaseous polytropes and stellar polytropes are nonlinearly dynamically stable with respect to the Euler-Poisson and Vlasov-Poisson systems respectively. It can be shown that a turning point of "temperature" $\beta$ appears for $n>3$ (Chavanis 2002b). Therefore, complete polytropes (and incomplete polytropes with high density

\footnotetext{
4 In fact, complete polytropes are equivalent by the homology theorem (Chandrasekhar 1942) but this is not the case in general for other stellar systems.
} 


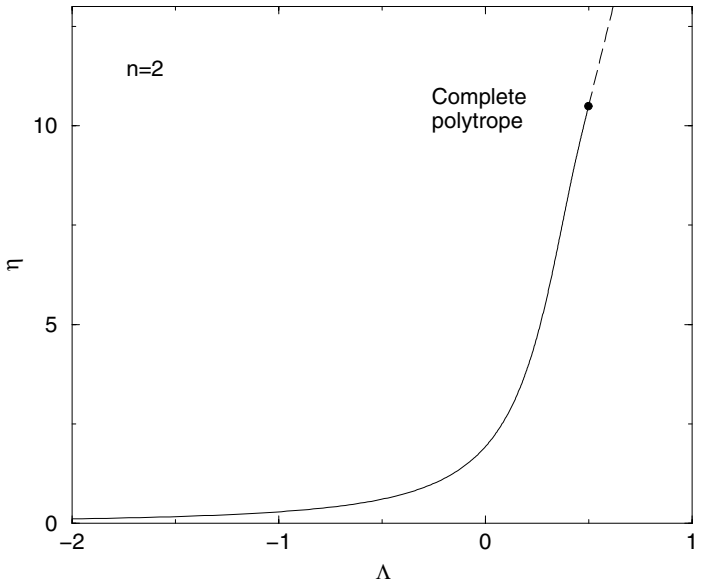

Fig. 6. Series of equilibria for polytropic spheres with index $n=2$ (see Chavanis 2002b; and Chavanis \& Sire 2004, for the details of the construction). In the thermodynamical analogy, this can be viewed as an effective caloric curve giving the effective normalized inverse temperature $\eta=M(G / n \Theta)^{\frac{n}{n-1}} / R^{\frac{n-3}{n-1}}$ (where $n \Theta=K(1+n) /(4 \pi)^{1 / n}$ is a polytropic temperature proportional to the polytropic constant $K$ ) as a function of the normalized energy $\Lambda=-E R / G M^{2}$. The parameter $\eta$ is a monotonic function of the Lagrange multiplier $\beta$ (through the relation $K=\beta^{-\left(1-\frac{3}{2 n}\right)}$ obtained from Eqs. (24) and (31) of Chavanis \& Sire 2004). Therefore, the curve $\eta-\Lambda$ is equivalent, with more convenient variables, to the curve $\beta(E)$ issued from the optimization problems (16) and (17). The first complete polytrope, with $\rho(R)=0$, is indicated by $(\bullet)$. It corresponds to the terminal point in the series of equilibria. The branch of complete polytropes with $R_{*}<R$ is indicated by a dashed line. For $n<3$, the series of equilibria is monotonic so that the "ensembles" are equivalent. Due to the correspondence that we have found, this means that for $n<3$ both stellar polytropes and polytropic stars are nonlinearly dynamically stable with respect to the Vlasov-Poisson and Euler-Poisson systems respectively.

contrast lying after the turning point of "temperature") cease to be minima of $F$ (at fixed mass) for $n>3$ (see Fig. 7). In that case, they lie in a region of the series of equilibria where the slope $\mathrm{d} E / \mathrm{d} T$ is negative. In the thermodynamical analogy, this is similar to a loss of canonical stability in a region of negative specific heats. Using the preceding results, we conclude that complete polytropic stars are nonlinearly dynamically stable with respect to the Euler-Poisson system for $n<3$ but they become linearly dynamically unstable for $n>3$. On the other hand, a turning point of energy $E$ appears for $n>5$ (see Fig. 8). Therefore, complete polytropes are maxima of $S$ (at fixed mass and energy) for $n<5$. Polytropes cease to be self-confined for $n>5$ and incomplete polytropes with high density contrast lying after the turning point of energy become unstable with respect to the Vlasov-Poisson system for $n>5$. This is similar to a loss of microcanonical stability in the thermodynamical analogy. In conclusion, stellar polytropes are nonlinearly dynamically stable with respect to the Vlasov-Poisson system for $3 / 2<n<5$ (they do not exist for $n<3 / 2$ ) and they become dynamically unstable for $n>5$ above a critical density contrast. On the other hand, gaseous polytropes are nonlinearly dynamically stable with respect to the Euler-Poisson system for $0<n<3$ and they become dynamically unstable for $n>3$ above a critical density contrast. For $3<n<5$,

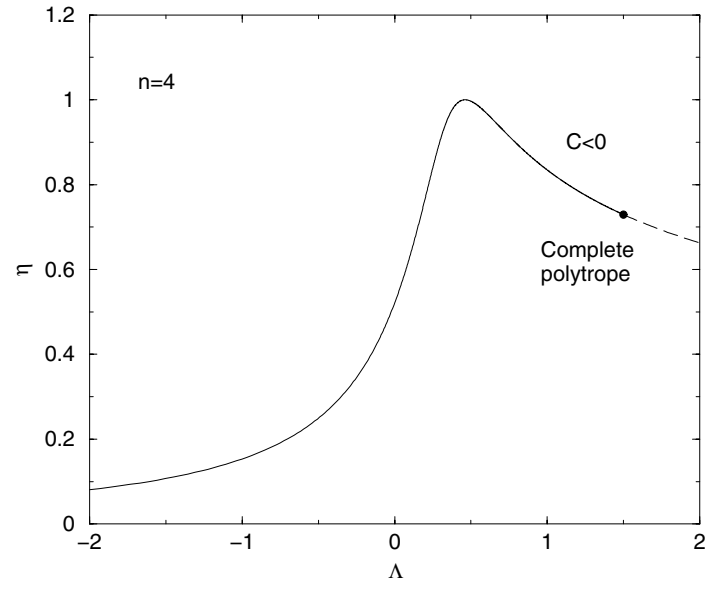

Fig. 7. Series of equilibria for polytropic spheres with index $n=4$. The first complete polytrope, with $R_{*}=R$, is indicated by $(\bullet)$ and the branch of complete polytropes with $R_{*}<R$ by a dashed line. Since they lie after the turning point of "temperature" (appearing for $n=3$ ) and before the turning point of energy (appearing for $n=5$ ), in the thermodynamical analogy, they are unstable in the "canonical" ensemble (saddle points of "free energy" $F$ at fixed $M$ ) but stable in the "microcanonical" ensemble (maxima of "entropy" $S$ at fixed $E$, $M)$. Due to the correspondance that we have found (see Sect. 4.2), these effective thermodynamical stability criteria mean in reality that stellar polytropes with $n=4$ are nonlinearly dynamically stable stationary solutions of the Vlasov-Poisson system but polytropic stars with $n=4$ are linearly dynamically unstable stationary solutions of the Euler-Poisson system. This is similar to a situation of "ensembles inequivalence" in thermodynamics. In particular, complete polytropes with $n=4$ lie in a region of effective negative specific heats $C<0$.

complete stellar polytropes are nonlinearly dynamically stable with respect to the Vlasov-Poisson system while corresponding polytropic stars are dynamically unstable with respect to the Euler-Poisson system. This is similar to a situation of ensembles inequivalence, in a region of negative specific heats, in thermodynamics. Of course, the dynamical stability of stellar polytropes and polytropic stars has been established for a long time in astrophysics but our method relying on the appearance of turning points in the series of equilibria and the interpretation of these results, and of the Antonov first law, in terms of a thermodynamical analogy and a situation of ensembles inequivalence is new and original. Furthermore, only linear dynamical stability is considered in general while the criteria (16) and (17) are criteria of nonlinear dynamical stability.

\subsection{The main point: correspondances}

In summary, we have the following analogy. In thermodynamics, if we plot the series of equilibria $\beta(E)$ we have the following result:

- After a turning point of temperature (or dimensionless parameter $\eta$ ), the system becomes canonically unstable (saddle point of free energy $F$ at fixed $M$ ). After a turning point of energy (or dimensionless parameter $\Lambda$ ), the system becomes microcanonically unstable (saddle point of entropy $S$ at fixed $E$, $M$ ). If the series of equilibria does not unwind, the system remains thermodynamically unstable after these turning points. 


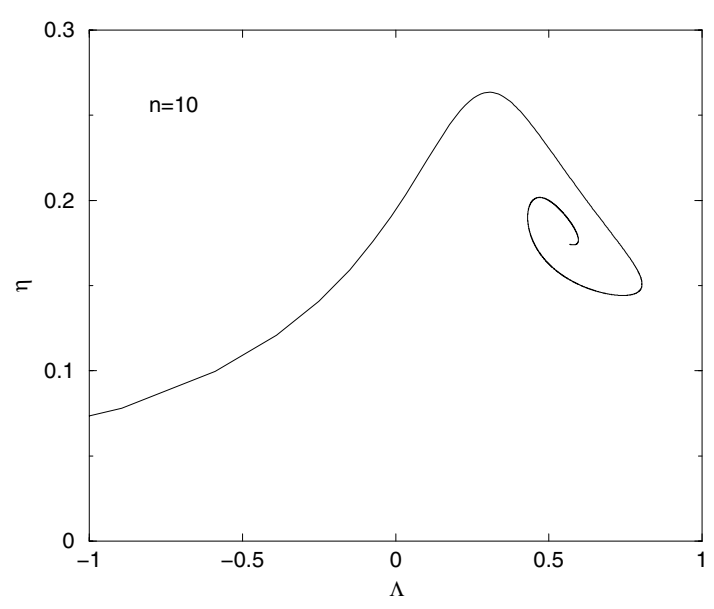

Fig. 8. Series of equilibria for polytropic spheres with index $n=10$. For $n>5$, self-gravitating polytropic spheres are unbounded and have infinite mass. Thus, equilibrium distributions only exist if the system is confined within a box. Box-confined gaseous polytropes cease to be stable with respect to the Euler-Poisson system after the first turning point of "temperature" $\eta$ and box-confined stellar polytropes cease to be stable with respect to the Vlasov-Poisson system after the first turning point of energy $\Lambda$. These stability criteria can be expressed in terms of the density contrast between center and edge which parameterizes the series of equilibria, like for isothermal spheres.

Before these turning points, the system is thermodynamically stable.

Concerning the nonlinear dynamical stability of collisionless stellar systems with $f=f(\epsilon), f^{\prime}(\epsilon)<0$ and corresponding barotropic stars with the same density distribution, if we plot the series of equilibria $\beta(E)$ we have the following result:

- After a turning point of "temperature" $\beta$ (or dimensionless parameter $\eta$ ), the barotropic gas becomes dynamically unstable with respect to the Euler-Poisson system. After a turning point of energy $E$ (or dimensionless parameter $\Lambda$ ), the stellar system becomes dynamically unstable with respect to the Vlasov-Poisson system. If the series of equilibria does not unwind, the system remains unstable after these turning points. Before these turning points, the system is nonlinearly dynamically stable.

Schematically, we have the correspondances:

(i) Nonlinear dynamical stability of barotropic stars $\leftrightarrow$ "canonical" criterion;

(ii) Nonlinear dynamical stability of spherical stellar systems $\leftrightarrow$ "microcanonical” criterion;

(iii) Nonlinear Antonov first law $\leftrightarrow$ canonical stability implies microcanonical stability.

\subsection{Other dimensions of space}

For completeness, we also show the series of equilibria for polytropic spheres in other dimensions of space $d$. These results can be of interest for mathematicians (see, e.g., Lemou et al. 2005). Furthermore, the problem is rich and interesting because it exhibits several critical dimensions with intriguing consequences concerning the particular dimension of our universe (see Chavanis 2004). The construction of these curves is

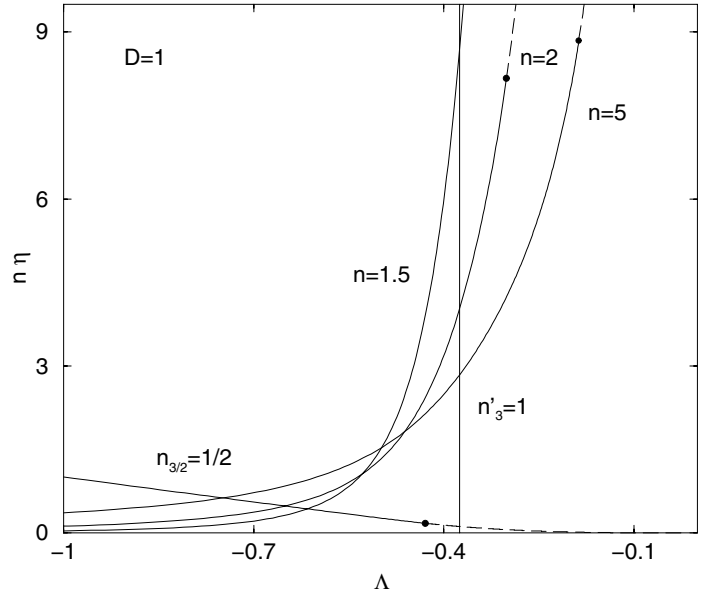

Fig. 9. Series of equilibria for polytropic spheres in $d=1$.

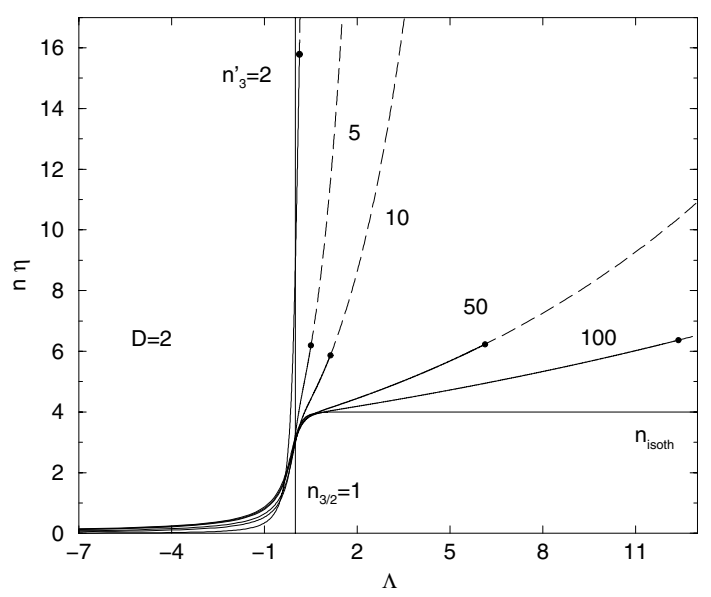

Fig. 10. Series of equilibria for polytropic spheres in $d=2$.

detailed in Chavanis \& Sire (2004) and some new curves are reported here in order to have a complete picture of the problem in parameter space $(d, n)$. We first recall that physical stellar polytropes (whose DF is a decreasing function of the energy) only exist for $n \geq n_{3 / 2} \equiv d / 2$ while gaseous polytropes can be considered a priori for any index (but we shall mostly consider the case $n \geq 0$ here). Furthermore, complete polytropes exist for all index if $d \leq 2$ and for $n<n_{5} \equiv(d+2) /(d-2)$ if $d>2$. Here, we only describe the stability of complete polytropes, i.e. the terminal point (bullet) in the series of equilibria.

For $d=1$ and $d=2$ the series of equilibria are monotonic (see Figs. 9 and 10) implying that both stellar polytropes with $n \geq n_{3 / 2}$ and polytropic stars with $n \geq 0$ are nonlinearly dynamically stable (the "ensembles" are equivalent).

For $2<d<4$, complete stellar polytropes exist for $n_{3 / 2}<n<n_{5}$ and they are nonlinearly dynamically stable (the turning point of energy appears for $n \geq n_{5}$ ). On the other hand, complete polytropic stars are nonlinearly dynamically stable for $0 \leq n<n_{3} \equiv d /(d-2)\left(n_{3}\right.$ is the index at which the turning point of "temperature" appears) and unstable for $n_{3} \leq n<n_{5}$ (see Fig. 11).

For $d=4$, complete stellar polytropes exist for $n_{3 / 2}=2<$ $n<n_{5}=3$ and they all have energy $E=0$ (see Fig. 12) as can be deduced from the results of Chavanis \& Sire (2004). 


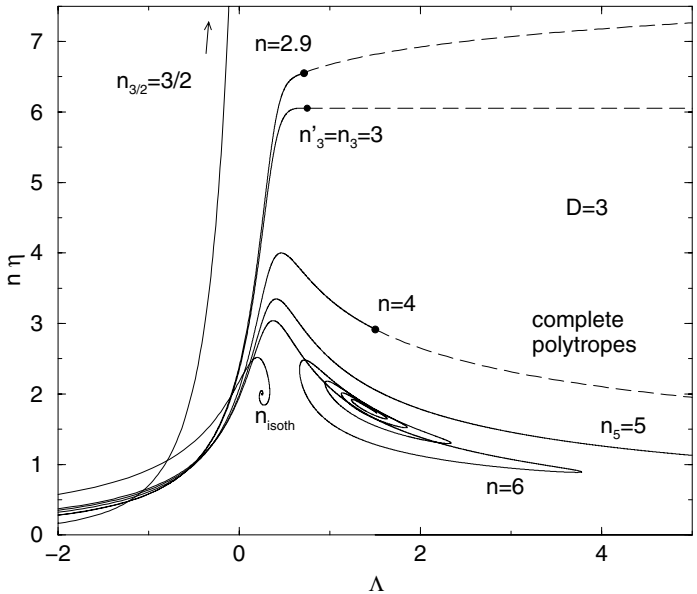

Fig. 11. Series of equilibria for polytropic spheres in $d=3$.

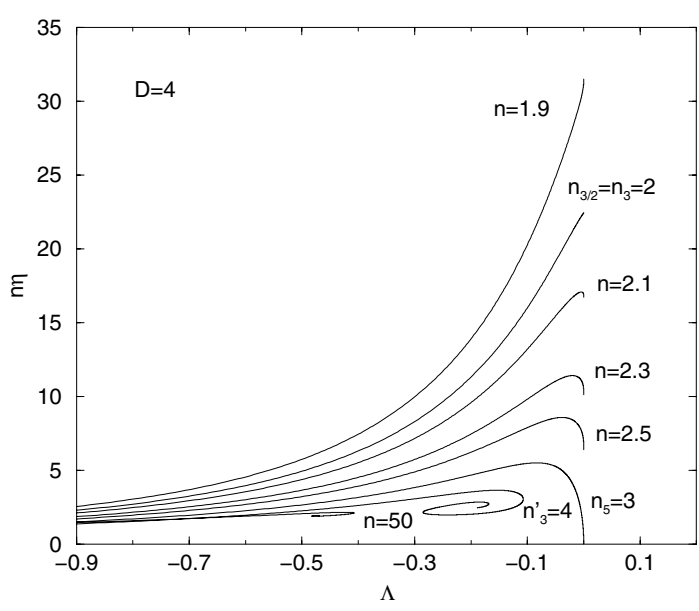

Fig. 12. Series of equilibria for polytropic spheres in $d=4$.

The series of equilibria has a vertical tangent at $E=0$ implying that complete stellar polytropes are marginally stable in $d=4$ (they lie precisely at the turning point of energy). This is consistent with the Virial theorem in $d=4$ for an isolated system (see Appendix C of Chavanis \& Sire 2005). On the other hand, complete polytropic stars are nonlinearly dynamically stable for $0 \leq n<n_{3}=2$ and unstable for $n_{3}=2 \leq n<n_{5}=3$ due to the presence of the turning point of "temperature".

For $d \geq 5$, there are no complete stellar polytropes with $n \geq n_{3 / 2}$ (the cross-over between $n_{5}$ and $n_{3 / 2}$ takes place for $d=$ $2(1+\sqrt{2}) \simeq 4.83 \ldots)$. On the other hand, complete polytropic stars are nonlinearly dynamically stable for $n<n_{3}$ and unstable for $n_{3} \leq n<n_{5}$ due to the presence of the turning point of "temperature" (see Fig. 13). For $n=n_{3 / 2}$ the series of equilibria makes angular points as explained in Appendix C of Chavanis $\&$ Sire (2004). For $n>n_{5}$, the series of equilibria forms a spiral whose sense of rotation changes for $n=n_{3 / 2}$. For $d \geq 10$ the situation slightly changes in the sense that the spiral shrinks to a point after an index $n_{-}$(see Chavanis \& Sire 2004). It is of interest to note that the spatial dimension $d=10$ is special in classical gravity (see Sire \& Chavanis 2002) as also found in theories of supergravity (e.g. Cariglia \& Mac Conamhna 2005). We do not know whether this is a coincidence of if it bears more physical meaning.

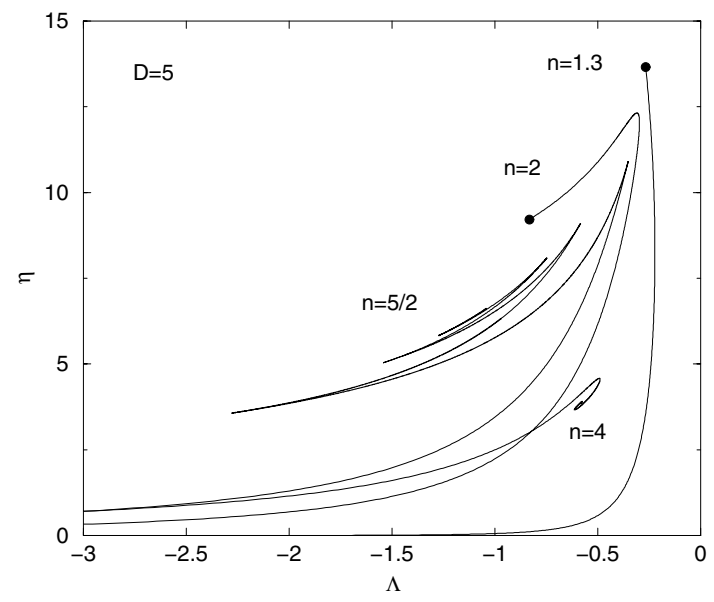

Fig. 13. Series of equilibria for polytropic spheres in $d=5$. For clarity, the curves have been multiplied by some factor to enter in the same figure.

We note finally that classical white dwarf stars are equivalent to polytropes with index $n_{3 / 2}=d / 2$ (Chavanis \& Sire 2004). Therefore, they are self-confined (complete) if $n_{3 / 2}<$ $n_{5}$, i.e. $d<2(1+\sqrt{2}) \simeq 4.83 \ldots$ and they are stable if $n_{3 / 2}<n_{3}$, i.e. $d<4$. For $d \geq 4$, quantum mechanics (Pauli exclusion principle) cannot stabilize matter against gravitational collapse (Chavanis 2004). Relativistic white dwarf stars are equivalent to polytropes with index $n_{3}^{\prime}=d$. Therefore, they are selfconfined (complete) if $n_{3}^{\prime}<n_{5}$, i.e. $d<\frac{1}{2}(3+\sqrt{17})=3.56 \ldots$ and they are stable if $n_{3}^{\prime}<n_{3}$, i.e. $d<3$. These results will be discussed more specifically in another paper (Chavanis 2006c).

\section{Phenomenology of violent relaxation}

In this section we show that the criterion of nonlinear dynamical stability (16) is remarkably consistent with the phenomenology of violent relaxation. Collisionless stellar systems like elliptical galaxies are believed to have achieved a steady state as a result of phase mixing and incomplete violent relaxation (Lynden-Bell 1967). During this process, the fine-grained distribution function $f(\boldsymbol{r}, \boldsymbol{v}, t)$ develops intermingled filaments at smaller and smaller scales so that it does not converge toward any steady distribution. However, if we locally average over these filaments, the coarse-grained distribution function $\bar{f}(\boldsymbol{r}, \boldsymbol{v}, t)$ is expected to converge towards a steady state $\bar{f}(\boldsymbol{r}, \boldsymbol{v})$ which is a stable stationary solution of the Vlasov equation. This is referred to as weak convergence in mathematics. Thus, the theory of violent relaxation explains how a collisionless stellar system, initially out-of-equilibrium, can reach a steady state of the Vlasov equation (on a coarsegrained scale) due to mean-field effects. Since this metaequilibrium state results from a complicated mixing process it is expected to be particularly robust and have nonlinear dynamical stability properties.

Now, Tremaine et al. (1986) have shown that the functionals $S[\bar{f}]=-\int C(\bar{f}) \mathrm{d} \boldsymbol{r} \mathrm{d} \boldsymbol{v}$, where $C$ is convex, calculated with the coarse-grained distribution function increase during violent relaxation in the sense that $S(t) \geq S(0)$ for $t \geq 0$ where it is assumed that the DF is not mixed at $t=0$, 
i.e. $\bar{f}(\boldsymbol{r}, \boldsymbol{v}, 0)=f(\boldsymbol{r}, \boldsymbol{v}, 0)$. For this reason, these functionals are called generalized $H$-functions. By contrast, the energy $E[\bar{f}]$ and the mass $M[\bar{f}]$ calculated with the coarse-grained DF are approximately conserved. The fact that $S[\bar{f}]$ increases at fixed $E[\bar{f}]$ and $M[\bar{f}]$ is similar to a generalized selective decay principle (for $-S$ ), like in $2 \mathrm{D}$ turbulence, which is due to phase mixing and coarse-graining. Phenomenologically, this may suggest that the system will reach a steady state $\bar{f}(\boldsymbol{r}, \boldsymbol{v})$ which maximizes a certain $H$-function (non-universal) at fixed mass and energy. This is consistent with the criterion of nonlinear dynamical stability (16) provided that we interpret the distribution function as the coarse-grained distribution function. Phase mixing and coarse-graining explain why we have to maximize a certain Casimir functional although the evolution is dissipationless and the Casimirs are conserved on the fine-grained scale ${ }^{5}$.

In reality, the problem is more complicated than that because (i) the $H$-functions do not increase necessarily monotonically with time (they are not Lyapunov functionals satisfying $\dot{S} \geq 0$ ); (ii) the system can converge toward a stationary solution of the Vlasov equation which does not depend on the energy $\epsilon$ alone, and thus which does not maximize an $H$-function at fixed $E, M$. For example, the velocity distribution of stars in elliptical galaxies is anisotropic and depends on the angular momentum $J=|\boldsymbol{r} \times \boldsymbol{v}|$ in addition to energy $\epsilon$. Furthermore, real stellar systems are in general not spherically symmetric so their DF does not only depend on $\epsilon$ and $J$. Therefore, more general stationary solutions of the Vlasov equation must be constructed in consistency with the Jeans theorem (Binney \& Tremaine 1987). However, their dynamical stability is more complicated to investigate.

More than constructing particular stationary solutions of the Vlasov equation in order to reproduce observations, it is a challenging issue to predict the distribution function of a galaxy resulting from incomplete violent relaxation. Lynden-Bell (1967) first addressed this question by developing a statistical theory of violent relaxation. He predicted a DF of the form $\bar{f}_{\mathrm{LB}}=\bar{f}_{\mathrm{LB}}(\epsilon)$ with $\bar{f}_{\mathrm{LB}}^{\prime}(\epsilon)<0$ which maximizes a certain $H$-function at fixed $E, M$ whose form depends on the initial conditions through the Casimirs (see Chavanis 2006a). In a dilute approximation which seems to apply to elliptical galaxies, the DF predicted by Lynden-Bell reduces to the Maxwell-Boltzmann distribution $f \sim \mathrm{e}^{-\beta \epsilon}$ which extremizes the Boltzmann entropy at fixed mass and energy. However, due to incomplete relaxation, this most mixed state is not reached in practice $^{6}$ and other stationary solutions of the Vlasov equation

\footnotetext{
5 The physical reason is the following. During mixing $D \bar{f} / D t \neq 0$ and the $H$-functions $S[\bar{f}]$ increase. Once it has mixed, $D \bar{f} / D t=0$ so that $\dot{S}[\bar{f}]=0$ meaning that the Casimirs of the coarse-grained DF are now conserved. If $\bar{f}(\boldsymbol{r}, \boldsymbol{v}, t)$ has been brought to a maximum $\bar{f}_{0}(\boldsymbol{r}, \boldsymbol{v})$ of a certain $H$-function (during violent relaxation) and since $S[\bar{f}]$ is conserved (after mixing), then $\bar{f}_{0}$ is a nonlinearly dynamically stable steady state of the Vlasov equation against coarse-grained perturbations, according to the criterion (16).

${ }^{6}$ This is obvious in the case of self-gravitating systems because the isothermal distribution coupled to the Poisson equation has infinite mass so it is ill-behaved mathematically. However, violent relaxation is in general incomplete also in systems for which the isothermal
}

can be reached instead. For example, ellipticals are well relaxed (in the sense of Lynden-Bell) in their inner region (leading to an isotropic isothermal core with density profile $\sim r^{-2}$ ) while they possess radially anisotropic envelopes (with density profile $\sim r^{-4}$ ). One success of the Lynden-Bell theory is to explain the isothermal cores of ellipticals without recourse to collisions.

The process of incomplete relaxation, corresponding to a lack of ergodicity (partial mixing), could be taken into account by developing dynamical models of violent relaxation as proposed in Chavanis et al. (1996) and Chavanis (1998) to understand what limits relaxation. Alternatively, in a series of papers, Bertin, Stiavelli and Trenti have proposed other ideas to account for incomplete violent relaxation. Stiavelli \& Bertin (1987) introduced an $f^{(\infty)}$ model of the form:

$f^{(\infty)}=A(-\epsilon)^{3 / 2} \mathrm{e}^{-a \epsilon-c J^{2} / 2}, \quad(\epsilon \leq 0)$

and $f=0$ for $\epsilon>0$, based on the possibility that the a priori probabilities of microstates are not equal due to kinetic constraints. This model reproduces many properties of ellipticals but it has the undesired feature of being "too isotropic". Then, they introduced another model based on a modification of the Lynden-Bell statistical theory. They considered the maximization of the Boltzmann entropy (in Lynden-Bell's sense) at fixed mass, energy and a third global quantity $Q=\int J^{v}|\epsilon|^{-3 v / 4} f \mathrm{~d} \boldsymbol{r d} v$ which is argued to be approximately conserved during violent relaxation (Trenti et al. 2005, have checked that $Q$ is reasonably well-conserved in numerical experiments of dissipationless galaxy formation). This variational principle results in a family of $f^{(v)}$ models:

$f^{(v)}=A \exp \left[-a \epsilon-d\left(\frac{J^{2}}{|\epsilon|^{3 / 2}}\right)^{v / 2}\right], \quad(\epsilon \leq 0)$

and $f=0$ for $\epsilon>0$. These models are able to fit products of $N$-body simulations over nine orders of magnitude in density and to reproduce the de Vaucouleur's $R^{1 / 4}$ law (or more general $R^{1 / n}$ laws) of ellipticals (Trenti et al. 2005). The introduction of additional constraints in the variational principle could be a way to take into account effects of incomplete violent relaxation. Bertin \& Trenti (2003) also showed that these models exhibit a sort of "gravothermal catastrophe" for collisionless stellar systems. They argue that this corresponds to a thermodynamical instability where internal collisionality is due not to stellar encounters (which are negligible because of the very long relaxation time) but to several dynamical causes making some relaxation proceed even at current epochs. According to the discussion of Sect. 4.1, the maximization of $S$ at fixed $E$, $M$ and $Q$ could also be viewed as a condition of nonlinear dynamical stability for the anisotropic DF (35) with respect to the Vlasov-Poisson system.

\section{Conclusion}

In this paper, we have shown that the dynamical (collisionless evolution) and the thermodynamical (collisional evolution)

distribution is well-behaved mathematically. This is the case, e.g., in 2D turbulence (Chavanis \& Sommeria 1998a; Brands et al. 1999). 
stability of stellar systems can be analyzed by similar methods. The thermodynamical stability of stellar systems has been investigated using optimization methods based on the maximization of the Boltzmann entropy at fixed mass and energy (Antonov 1962; Lynden-Bell \& Wood 1968; Katz 1978; Padmanabhan 1990; Chavanis 2002a). In contrast, the dynamical stability of collisionless stellar systems described by the Vlasov-Poisson system has been customarily analyzed by linearizing the Vlasov equation around a steady state and studying the resulting eigenvalue equation (Binney \& Tremaine 1987). We have approached the dynamical stability problem by a different method, through the maximization of a Casimir functional (or $H$-function) at fixed mass and energy. This provides a condition of nonlinear dynamical stability. Furthermore, this allows us to develop a close formal analogy with the thermodynamical problem. Due to this analogy, we can use methods similar to those introduced in thermodynamics (series of equilibria, Legendre transforms, Poincaré's turning point argument, ...) to settle the nonlinear dynamical stability of a spherical stellar system. Thus, the existence of turning points of temperature and energy which respectively mark the onset of canonical and microcanonical instability in thermodynamics also mark the onset of instability for barotropic stars and spherical stellar systems in the dynamical stability problem with respect to the Euler and Vlasov equations. For example, when we plot the series of equilibria of polytropes, a turning point of "temperature" appears for $n \geq 3$ and a turning point of energy appears for $n \geq 5$; these are precisely the indices marking the dynamical instability thresholds of polytropic stars and stellar polytropes respectively. These indices had been previously found by other authors using very different arguments (see Binney \& Tremaine 1987). In this paper, we have shown that the nonlinear Antonov first law could be viewed as a manifestation of "ensembles inequivalence" in self-gravitating systems. This is an original interpretation of this law. This thermodynamical analogy allows us to unify two types of studies (dynamical and thermodynamical) that had been previously approached by very different methods.

There are, however, some limitations in our approach: (i) Our criteria of nonlinear dynamical stability only apply to spherical stellar systems described by a DF of the form $f=$ $f(\epsilon)$ with $f^{\prime}(\epsilon)<0$. It is not clear how they can be extended to more general steady distributions of the Vlasov equation satisfying the Jeans theorem. However, in the case of anisotropic stellar systems, we have proposed (see Chavanis 2003a) considering the maximization of an $H$-function at fixed mass, energy and $\boldsymbol{L}_{2}=\int f(\boldsymbol{r} \times \boldsymbol{v})^{2} \mathrm{~d} \boldsymbol{r} \mathrm{d} \boldsymbol{v}$ which could be viewed as an adiabatic invariant (it is not strictly conserved by the Vlasov equation, but it can be approximately conserved in some situations $)^{7}$. The extremization problem leads to a DF of the form $f=f\left(\epsilon_{\mathrm{a}}\right)$ with $\epsilon=\epsilon+\frac{J^{2}}{2 r_{\mathrm{a}}}$ and $f^{\prime}\left(\epsilon_{\mathrm{a}}\right)<0\left(r_{\mathrm{a}}\right.$ is the anisotropy radius and $J=|\boldsymbol{r} \times \boldsymbol{v}|$ the angular momentum). Furthermore, the condition of maximum is probably related to a form of nonlinear dynamical stability. These intuitive results have to be made

\footnotetext{
7 This idea turns out to be similar to that introduced earlier by Stiavelli \& Bertin (1987) with the conservation of an additional quantity $Q=\int J^{v}|\epsilon|^{-3 v / 4} f \mathrm{~d} \boldsymbol{r} \mathrm{d} v$, see Sect. 5 .
}

more rigorous. (ii) To use the turning point method, we have been led to confine the system within a box. Physically, only the last point in the series of equilibria that corresponds to a selfconfined structure unaffected by the box (the density $\rho(R)=0$ vanishes on the boundary) is physically relevant (see, e.g., the complete polytrope in Fig. 7). However, the whole curve is needed to determine whether this state lies before or after the turning point of "temperature" $\beta$ or energy $E$. Certainly, the dynamical stability of self-confined systems (with a compact support) could be settled by other means, without plotting the whole series of equilibria for box-confined configurations (see Rein 2005). However, in that case we miss the interesting analogy with the thermodynamical problem that we have found. Furthermore, there are situations where the box is necessary. Technically, the box is needed when the system can never be self-confined. This is the case for isothermal stellar systems and polytropes with index $n \geq 5$. One justification of the box in that case is to delimitate the region of space where the distribution function of the above type holds ${ }^{8}$. For example, the box could mimic the effect of a tidal radius in the case of globular clusters. On the other hand, we have found that the dynamical and thermodynamical methods developed in astrophysics can have applications for other systems with long-range interactions like two-dimensional vortices in hydrodynamics (Chavanis 2002c), bacterial populations in biology (Chavanis et al. 2004) and the HMF model (Chavanis et al. 2005). For these systems, there are situations where the introduction of a material box is physically relevant. The general study of the stability problems, even in idealized situations of box-confined configurations, is not just academic but can have useful applications in unexpected areas. Therefore, it is important to consider the problem in full generality even if we sacrifice for direct astrophysical applications. Finally, instead of introducing a material box, we can use a confinement in energy space by using truncated distribution functions like the Michie-King model. In that case the method of linear series of equilibria still holds as shown by Katz (1980) who analyzed different truncated models and determined the limit of stability for each of them. Interestingly, he found that the limits of stability are not too sensitive to the form of confinement. Note that according to the criterion (16), the stability limits found by Katz (1980) can be interpreted either as limits of thermodynamical stability or as limits of nonlinear dynamical stability with respect to the Vlasov-Poisson system.

In conclusion, the method of linear series of equilibria is very powerful and allows us to determine the limits of stability for various models. In particular, one goal of this paper was to show that this method can be used for nonlinear dynamical stability problems, not just for thermodynamical stability problems. This offers a connection between different types of models: isothermal stellar systems, self-gravitating fermions, polytropes, Michie-King models (truncated isothermals) etc. Furthermore, they allow us to determine their stability limits very easily from a purely graphical construction: we just

\footnotetext{
${ }^{8}$ Instead of a rigid box, we could consider a system in contact with an external medium imposing a constant pressure $P$. In that case, the method of linear series of equilibria still works in the so-called isobaric ensemble (see Chavanis 2003a).
} 
need to plot the series of equilibria $\beta(E)$ (this only demands that we solve the first order variational problem) and then check whether the configuration of interest lies before or after the turning point of temperature or energy in the series of equilibria. In thermodynamics, the first turning point of temperature implies a loss of canonical stability and the first turning point of energy a loss of microcanonical stability. For the nonlinear dynamical stability problem, the first turning point of "temperature" (in the sense of our thermodynamical analogy) implies a loss of nonlinear dynamical stability for a barotropic star with respect to the Euler-Poisson system and the first turning point of energy a loss of nonlinear dynamical stability for a spherical stellar system with respect to the Vlasov-Poisson system.

Note added in proof. After final submission of the paper, the following references were discovered which are also relevant to our subject: Hjorth, J. 1994, ApJ, 424, 106 and Ducomet, B. \& Zlotnik, A. 2005, Appl. Math. Lett., 18, 1190.

\section{Appendix A: The point of marginal stability}

Let us consider a stationary solution of the Euler-Poisson system (1)-(3) satisfying $\boldsymbol{u}=\mathbf{0}$ and the condition of hydrostatic equilibrium (6). The linearized Euler-Poisson equations around this solution are

$\frac{\partial \delta \rho}{\partial t}+\nabla \cdot(\rho \delta \boldsymbol{u})=0$

$\rho \frac{\partial \delta \boldsymbol{u}}{\partial t}=-\nabla\left(p^{\prime}(\rho) \delta \rho\right)-\rho \nabla \delta \Phi-\delta \rho \nabla \Phi$

$\Delta \delta \Phi=4 \pi G \delta \rho$.

Considering spherically symmetric systems and writing the evolution of the perturbation as $\delta \rho \sim \mathrm{e}^{\sigma t}$, we get

$\sigma \delta \rho+\frac{1}{r^{2}} \frac{\mathrm{d}}{\mathrm{d} r}\left(r^{2} \rho \delta u\right)=0$

$\sigma \rho \delta u=-\frac{\mathrm{d}}{\mathrm{d} r}\left(p^{\prime}(\rho) \delta \rho\right)-\rho \frac{\mathrm{d} \delta \Phi}{\mathrm{d} r}-\delta \rho \frac{\mathrm{d} \Phi}{\mathrm{d} r}$

$\frac{1}{r^{2}} \frac{\mathrm{d}}{\mathrm{d} r}\left(r^{2} \frac{\mathrm{d} \delta \Phi}{\mathrm{d} r}\right)=4 \pi G \delta \rho$.

We write

$\delta \rho=\frac{1}{4 \pi r^{2}} \frac{\mathrm{d} q}{\mathrm{~d} r}$

where $q(r) \equiv \delta M(r)=\int_{0}^{r} \delta \rho\left(r^{\prime}\right) 4 \pi r^{\prime 2} \mathrm{~d} r^{\prime}$ denotes the mass perturbation within the sphere of radius $r$. Using the Gauss theo$\operatorname{rem} \mathrm{d} \delta \Phi / \mathrm{d} r=G q / r^{2}$, we see that the Poisson Eq. (A.6) is automatically satisfied. On the other hand, the continuity Eq. (A.4) leads to

$\delta u=-\frac{\sigma}{4 \pi \rho r^{2}} q$,

where we have used $q(0)=\delta u(0)=0$ to eliminate the constant of integration. Finally, substituting Eqs. (A.7) and (A.8) in Eq. (A.5) and using the Gauss theorem and the condition of hydrostatic equilibrium $\mathrm{d} p / \mathrm{d} r=-\rho \mathrm{d} \Phi / \mathrm{d} r$ we finally obtain

$\frac{\mathrm{d}}{\mathrm{d} r}\left(\frac{p^{\prime}(\rho)}{4 \pi \rho r^{2}} \frac{\mathrm{d} q}{\mathrm{~d} r}\right)+\frac{G q}{r^{2}}=\frac{\sigma^{2}}{4 \pi \rho r^{2}} q$.

This is the required equation of pulsations for a self-gravitating barotropic gas. It is equivalent to the Eddington (1926) equation of pulsations albeit written in a different form (see Chavanis \& Sire 2005). For isothermal and polytropic equations of state, we recover the equations studied in Chavanis (2002a,b, 2003a). It can be shown that the eigenvalues $\sigma^{2}$ are real. Therefore, the condition of stability is $\sigma^{2}<0$ corresponding to an oscillatory behavior of the perturbation. The condition of marginal stability is $\sigma=0$.

Let us now consider the minimization of the energy functional (4). The second order variations can be written

$\delta^{2} \mathcal{W}=\frac{1}{2} \int_{0}^{R} \frac{\mathrm{d} q}{\mathrm{~d} r} \delta \Phi \mathrm{d} r+\int_{0}^{R} \frac{p^{\prime}(\rho)}{8 \pi \rho r^{2}}\left(\frac{\mathrm{d} q}{\mathrm{~d} r}\right)^{2} \mathrm{~d} r$.

Integrating by parts and using $q(0)=q(R)=0$, we obtain

$\delta^{2} \mathcal{W}=-\frac{1}{2} \int_{0}^{R} q \frac{\mathrm{d} \delta \Phi}{\mathrm{d} r} \mathrm{~d} r-\int_{0}^{R} \frac{\mathrm{d}}{\mathrm{d} r}\left(\frac{p^{\prime}(\rho)}{8 \pi \rho r^{2}} \frac{\mathrm{d} q}{\mathrm{~d} r}\right) q \mathrm{~d} r$.

Then, using the Gauss theorem, we get

$\delta^{2} \mathcal{W}=-\frac{1}{2} \int_{0}^{R}\left[\frac{\mathrm{d}}{\mathrm{d} r}\left(\frac{p^{\prime}(\rho)}{4 \pi \rho r^{2}} \frac{\mathrm{d} q}{\mathrm{~d} r}\right)+\frac{G q}{r^{2}}\right] q \mathrm{~d} r$.

We are led therefore to consider the eigenvalue problem

$\left[\frac{\mathrm{d}}{\mathrm{d} r}\left(\frac{p^{\prime}(\rho)}{4 \pi \rho r^{2}} \frac{\mathrm{d}}{\mathrm{d} r}\right)+\frac{G}{r^{2}}\right] q_{\lambda}(r)=\lambda q_{\lambda}(r)$

If all the eigenvalues $\lambda$ are negative, then $\delta^{2} \mathcal{W}>0$ and the configuration is a minimum of $\mathcal{W}$ at fixed mass. This implies that it is nonlinearly dynamically stable. If at least one eigenvalue $\lambda$ is positive, the configuration is a saddle point of $\mathcal{W}$ and the stability criterion is not satisfied. The marginal case is when the largest eignenvalue $\lambda$ is equal to zero. In that case, Eqs. (A.9) and (A.13) coincide. This implies that the point in the series of equilibria where the system becomes linearly unstable $(\sigma=0)$ coincides with the point where it ceases to be a minimum of $\mathcal{W}$, i.e. $\lambda=0$. Therefore, the onset of linear and nonlinear dynamical stability coincides, as previously found in the particular cases of isothermal and polytropic distributions (Chavanis 2002a,b, 2003a).

\section{Appendix B: Passage from $F[f]$ to $F[\rho]$}

The optimal distribution function $f_{*}$ which minimizes $F[f]$ at fixed density profile $\rho(\boldsymbol{r})$ is determined by $C^{\prime}\left(f_{*}\right)=-\beta\left(v^{2} / 2+\right.$ $\lambda(\boldsymbol{r})$ ). Since $C$ is convex, this relation can be inversed to give

$f_{*}(\boldsymbol{r}, \boldsymbol{v})=F\left[\beta\left(\frac{v^{2}}{2}+\lambda(\boldsymbol{r})\right)\right]$, 
where $F(x)=\left(C^{\prime}\right)^{-1}(-x)$. The density $\rho=\int f_{*} \mathrm{~d} v$ and the pressure $p=\frac{1}{d} \int f_{*} v^{2} \mathrm{~d} \boldsymbol{v}$ (in $d$ dimensions) can then be expressed as

$\rho=\frac{1}{\beta^{d / 2}} g(\beta \lambda), \quad p=\frac{1}{\beta^{\frac{d+2}{2}}} h(\beta \lambda)$,

with

$g(x)=2^{\frac{d-2}{2}} S_{d} \int_{0}^{+\infty} F(x+t) t^{\frac{d-2}{2}} \mathrm{~d} t$,

$h(x)=\frac{1}{d} 2^{\frac{d}{2}} S_{d} \int_{0}^{+\infty} F(x+t) t^{\frac{d}{2}} \mathrm{~d} t$,

where $S_{d}$ is the surface of a unit sphere in $d$ dimensions. Eliminating $\lambda$ between the foregoing expressions, we find that $p=p(\rho)$ where the barotropic equation of state is entirely specified by $C(f)$. We can now express $F[f]=E[f]-T S[f]$ as a functional of $\rho$ by writing $F[\rho]=F\left[f_{*}\right]$. The energy is simply given by

$E=\frac{d}{2} \int p \mathrm{~d} \boldsymbol{r}+\frac{1}{2} \int \rho \Phi \mathrm{d} \boldsymbol{r}$.

On the other hand, the Casimir (15) can be written

$S=-\frac{2^{\frac{d-2}{2}} S_{d}}{\beta^{d / 2}} \int \mathrm{d} \boldsymbol{r} \int_{0}^{+\infty} C[F(t+\beta \lambda)] t^{\frac{d-2}{2}} \mathrm{~d} t$.

Integrating by parts and using $C^{\prime}[F(x)]=-x$, we find that

$S=-\frac{2^{d / 2} S_{d}}{d \beta^{d / 2}} \int \mathrm{d} \boldsymbol{r} \int_{0}^{+\infty} F^{\prime}(t+\beta \lambda)(t+\beta \lambda) t^{d / 2} \mathrm{~d} t$.

Integrating by parts one more time and using Eqs. (B.2), (B.3) and (B.4), we finally obtain

$S=\frac{d+2}{2} \beta \int p \mathrm{~d} \boldsymbol{r}+\beta \int \lambda \rho \mathrm{d} \boldsymbol{r}$.

Collecting all the previous results, we get

$F[\rho]=-\int \rho\left(\lambda+\frac{p}{\rho}\right) \mathrm{d} \boldsymbol{r}+\frac{1}{2} \int \rho \Phi \mathrm{d} \boldsymbol{r}$.

Finally, using the relation $h^{\prime}(x)=-g(x)$ obtained from Eqs. (B.3) and (B.4) by a simple integration by parts, it is easy to check that Eq. (B.2) implies

$\lambda^{\prime}(\rho)=-\frac{p^{\prime}(\rho)}{\rho}$,

so that

$\lambda+\frac{p}{\rho}=-\int^{\rho} \frac{p\left(\rho^{\prime}\right)}{\rho^{\prime 2}} \mathrm{~d} \rho^{\prime}$.

Thus,

$F[\rho]=\int \rho \int_{0}^{\rho} \frac{p\left(\rho^{\prime}\right)}{\rho^{\prime 2}} \mathrm{~d} \rho^{\prime} \mathrm{d} \boldsymbol{r}+\frac{1}{2} \int \rho \Phi \mathrm{d} \boldsymbol{r}$.

\section{References}

Antonov, V. A. 1960, Astr. Zh, 37, 918

Antonov, V. A. 1962, Vest. Leningr. Gos. Univ. 7, 135

Arnold, V. 1966, Izv. Vyssh. Uchebn. Zaved Matematika, 54, 3

Bertin, G., \& Trenti, M. 2003, ApJ, 584, 729

Binney, J., \& Tremaine, S. 1987, Galactic Dynamics, Princeton Series in Astrophysics
Boghosian, B. M. 1996, Phys. Rev. E, 53, 4754

Bouchet, F., \& Barré, J. 2005, J. Stat. Phys., 118, 1073

Brands, H., Chavanis, P. H., Sommeria, J., \& Pasmanter, R. 1999, Phys. Fluids, 11, 3465

Chandrasekhar, S. 1942, An Introduction to the Theory of Stellar Structure (Dover)

Cariglia, M., \& Mac Conamhna, O. 2005, PRL, 94, 161601

Chavanis, P. H. 1998, MNRAS, 300, 981

Chavanis, P. H. 2002a, A\&A, 381, 340

Chavanis, P. H. 2002b, A\&A, 386, 732

Chavanis, P. H. 2002c, in Dynamics and thermodynamics of systems with long range interactions, ed. T. Dauxois, S. Ruffo, E. Arimondo, \& M. Wilkens, Lecture Notes in Physics (Springer) [cond-mat/0212223]

Chavanis, P. H. 2002d, PRE, 65, 056123

Chavanis, P. H. 2003a, A\&A, 401, 15

Chavanis, P. H. 2003b, PRE, 68, 036108

Chavanis, P. H. 2004, PRE, 69, 6126

Chavanis, P. H. 2005a, A\&A, 432, 117

Chavanis, P. H. 2005b [cond-mat/0509726]

Chavanis, P. H. 2006a, Physica A, 359, 177

Chavanis, P. H. 2006b, Physica A, 361, 81

Chavanis, P. H. 2006c, [arXiv: astro-ph/0604012]

Chavanis, P. H., \& Rieutord, M. 2003, A\&A, 412, 1

Chavanis, P. H., \& Sire, C. 2004, PRE, 69, 016116

Chavanis, P. H., \& Sire, C. 2005 [cond-mat/0504718]

Chavanis, P. H., \& Sommeria, J. 1998a, JFM, 356, 259

Chavanis, P. H., \& Sommeria, J. 1998b, MNRAS, 296, 569

Chavanis, P. H., Rosier, C., \& Sire, C. 2002, PRE, 66, 036105

Chavanis, P. H., Ribot, M., Rosier, C., \& Sire, C. 2004, Banach Center Publ., 66, 103 [cond-mat/0407386]

Chavanis, P. H., Sommeria, J., \& Robert, R. 1996, ApJ, 471, 385

Chavanis, P. H., Vatteville, J., \& Bouchet, F. 2005, EPJB, 46, 61

Eddington, A. S. 1926, Internal Constitution of the Stars (Cambridge University Press)

Ellis, R., Haven, K., \& Turkington, B. 2000, J. Stat. Phys., 101, 999

Ellis, R., Haven, K., \& Turkington, B. 2002, Nonlinearity, 15, 239

Guo, Y., \& Rein, G. 1999, Arch. Rational Mech. Anal., 147, 225

Guo, Y., \& Rein, G. 2001, Commun. Math. Phys., 219, 607

Holm, D. D., Marsden, J. E., Ratiu, T., \& Weinstein, A. 1985, Phys. Rep., 123, 1

Katz, J. 1978, MNRAS, 183, 765

Katz, J. 1980, MNRAS, 190, 497

Kull, A., Treumann, R. A., \& Böhringer, H. 1997, ApJ, 484, 58

Latora, V., Rapisarda, A., \& Tsallis, C. 2002, Physica A, 305, 129

Lemou, M., Méhats, F., \& Raphael, P. 2005, CRAS, 341, 269

Lynden-Bell, D. 1967, MNRAS, 136, 101

Lynden-Bell, D., \& Lynden-Bell, R. M. 1977, MNRAS, 181, 405

Lynden-Bell, D., \& Wood, R. 1968, MNRAS, 138, 495

May, A., \& van Albada, T. S. 1984, MNRAS, 209, 15

Ogorodnikov, K. F. 1965, Dynamics of stellar systems (Pergamon)

Padmanabhan, T. 1990, Phys. Rep., 188, 285

Rein, G. 2003, Arch. Rational Mech. Anal., 168, 115

Rein, G. 2005 [arXiv: astro-ph/0501541]

Sire, C., \& Chavanis, P. H. 2002, PRE, 66, 046133

Stiavelli, M., \& Bertin, G. 1987, MNRAS, 229, 61

Taruya, A., \& Sakagami, M. 2003, Physica A, 322, 285

Thirring, W. 1970, Z. Phys., 235, 339

Tremaine, S., Hénon, M., \& Lynden-Bell, D. 1986, MNRAS, 219, 285

Trenti, M., Bertin, G., \& van Albada, T. S. 2005, A\&A, 433, 57

Tsallis, C. 1988, J. Stat. Phys., 52, 479 